\title{
Cycles on curves and Jacobians: a tale of two tautological rings
}

\author{
Qizheng Yin
}

\begin{abstract}
We connect two notions of tautological ring: one for the moduli space of curves (after Mumford, Faber, etc.), and the other for the Jacobian of a curve (after Beauville, Polishchuk, etc.). The motivic Lefschetz decomposition on the Jacobian side produces relations between tautological classes, leading to results about Faber's Gorenstein conjecture on the curve side. We also relate certain Gorenstein properties on both sides and verify them for small genera. Further, we raise the question whether all tautological relations are motivic, giving a possible explanation why the Gorenstein properties may not hold.
\end{abstract}

\section{Introduction}

Tautological classes are classes of algebraic cycles carrying certain geometric information. They form a ring called the tautological ring. Classically there are two such notions of tautological ring: one introduced by Mumford [Mum83] for the moduli space of curves, and the other by Beauville [Bea04] for the Jacobian of a curve (and modulo algebraic equivalence).

1.1. Curve side. Denote by $\mathscr{M}_{g}$ the moduli space of smooth curves of genus $g$, and by $p: \mathscr{C}_{g} \rightarrow$ $\mathscr{M}_{g}$ the universal curve. Consider the classes $\kappa_{i}=p_{*}\left(K^{i+1}\right)$, with $K$ the relative canonical divisor. The tautological ring $\mathscr{R}\left(\mathscr{M}_{g}\right)$ is defined to be the $\mathbb{Q}$-subalgebra of the Chow ring $\mathrm{CH}\left(\mathscr{M}_{g}\right)$ (with $\mathbb{Q}$-coefficients) generated by $\left\{\kappa_{i}\right\}$.

The study of $\mathscr{R}\left(\mathscr{M}_{g}\right)$ is partly motivated by Faber's Gorenstein conjecture [Fab99], which predicts that $\mathscr{R}\left(\mathscr{M}_{g}\right)$ is a Gorenstein ring with socle in degree $g-2$. The difficulty is to find enough relations between the generators: starting from $g=24$, all known methods have failed to do so. On the other hand, apart from numerical evidence, there has been no convincing reason for or against the conjecture.

One may consider variants of $\mathscr{R}\left(\mathscr{M}_{g}\right)$ for the universal curve $\mathscr{C}_{g}$ and its relative powers $\mathscr{C}_{g}^{n}$. There is also a pointed version, namely over the moduli of smooth pointed curves $\mathscr{M}_{g, 1}$. In all cases one can ask about the corresponding Gorenstein properties.

Received 15 July 2014, accepted in final form 26 June 2015.

2010 Mathematics Subject Classification 14C25, 14H10, $14 \mathrm{H} 40$.

Keywords: moduli of curves, Jacobian, symmetric power, tautological ring, Faber conjectures.

This journal is (c) Foundation Compositio Mathematica 2016. This article is distributed with Open Access under the terms of the Creative Commons Attribution Non-Commercial License, which permits non-commercial reuse, distribution, and reproduction in any medium, provided that the original work is properly cited. For commercial re-use, please contact the Foundation Compositio Mathematica.

The author was supported by the grant ERC-2012-AdG-320368-MCSK. 
1.2. Jacobian side. Let $J$ be the Jacobian of a smooth pointed curve $\left(C, x_{0}\right)$. The point $x_{0}$ induces an embedding $C \hookrightarrow J$. Since $J$ is an abelian variety, its Chow ring carries a second ring structure and also the action of the multiplication by $N \in \mathbb{Z}$. The tautological ring $\mathscr{T}(J)$ is defined to be the smallest $\mathbb{Q}$-subspace of $\mathrm{CH}(J)$ (with $\mathbb{Q}$-coefficients) containing the curve class $[C]$ and stable under both ring structures as well as the multiplication by $N$. One can prove that $\mathscr{T}(J)$ is finitely generated (with respect to the intersection product) and write down an explicit set of generators.

In a series of papers ([Pol05, Pol07b, Pol07a], etc.), Polishchuk applied powerful tools to the study of $\mathscr{T}(J)$, such as the Beauville decomposition, the Fourier transform and the Lefschetz decomposition (or the $\mathfrak{s l}_{2}$-action). Notably, he used the third tool to construct relations between the generators. For the generic curve (and modulo algebraic equivalence), he conjectured that they give all the relations (see [Pol05, Introduction]).

Polishchuk's approach also brings a motivic touch to the subject. The hidden background is the so-called motivic Lefschetz decomposition, and the relations obtained this way are simply dictated by the motive of $J$.

1.3. Main results. Our first result extends the Jacobian side to the relative (or universal) setting. Denote by $\mathscr{J}_{g, 1}$ the universal Jacobian over $\mathscr{M}_{g, 1}$. The tautological ring $\mathscr{T}\left(\mathscr{J}_{g, 1}\right)$ is defined similarly. By studying the $\mathfrak{s l}_{2}$-action on $\mathscr{T}\left(\mathscr{J}_{g, 1}\right)$, we obtain the following statement (Theorem 4.5).

TheOREM 1. The ring $\mathscr{T}\left(\mathscr{J}_{g, 1}\right)$ has an explicit finite set of generators (with respect to the intersection product). The $\mathfrak{s l}_{2}$-action on $\mathscr{T}\left(\mathscr{J}_{g, 1}\right)$ can also be described explicitly in terms of the generators.

Our second result connects the tautological rings on both sides. Denote by $\mathscr{C}_{g, 1}$ the universal curve over $\mathscr{M}_{g, 1}$, and by $\mathscr{C}_{g, 1}^{n}$ (respectively, $\mathscr{C}_{g, 1}^{[n]}$ ) its $n$th power (respectively, symmetric power) relative to $\mathscr{M}_{g, 1}$. The tautological rings $\mathscr{R}\left(\mathscr{C}_{g, 1}^{n}\right)$ and $\mathscr{R}\left(\mathscr{C}_{g, 1}^{[n]}\right)$ are defined accordingly. Further, one passes to the limit $\mathscr{C}_{g, 1}^{[\infty]}=\lim _{\longrightarrow} \mathscr{C}_{g, 1}^{[n]}$ and gets the tautological ring $\mathscr{R}\left(\mathscr{C}_{g, 1}^{[\infty]}\right)$. Then we have the following comparison (Corollary 4.7 and Theorem 6.4).

TheOREM 2. The ring $\mathscr{R}\left(\mathscr{C}_{g, 1}^{[\infty]}\right)$ is a polynomial algebra over $\mathscr{T}\left(\mathscr{J}_{g, 1}\right)$. In particular, the ring $\mathscr{R}\left(\mathscr{M}_{g, 1}\right)$ is a $\mathbb{Q}$-subalgebra of $\mathscr{T}\left(\mathscr{J}_{g, 1}\right)$.

Following Polishchuk, we use the $\mathfrak{s l}_{2}$-action on $\mathscr{T}\left(\mathscr{J}_{g, 1}\right)$ to produce tautological relations. With these relations, we are able to confirm that $\mathscr{R}\left(\mathscr{M}_{g, 1}\right)$ (respectively, $\mathscr{R}\left(\mathscr{M}_{g}\right)$ ) is Gorenstein for $g \leqslant 19$ (respectively, $g \leqslant 23$ ). As far as our computation goes, we seem to recover all the Faber-Zagier relations. Moreover, the socle condition for $\mathscr{R}\left(\mathscr{C}_{g, 1}^{[n]}\right)$ allows us to formulate the corresponding Gorenstein property for $\mathscr{T}\left(\mathscr{J}_{g, 1}\right)$, which is again confirmed for $g \leqslant 7$. Our third result is an equivalence of Gorenstein properties (Theorem 7.15).

TheOREM 3. The ring $\mathscr{T}\left(\mathscr{J}_{g, 1}\right)$ is Gorenstein if and only if $\mathscr{R}\left(\mathscr{C}_{g, 1}^{[n]}\right)$ is Gorenstein for all $n \geqslant 0$.

It follows that $\mathscr{R}\left(\mathscr{C}_{g, 1}^{[n]}\right)$ (respectively, $\mathscr{R}\left(\mathscr{C}_{g}^{[n]}\right)$ ) is Gorenstein for $g \leqslant 7$ and for all $n \geqslant 0$.

1.4. We also say a few words on the value of this paper and how it relates to other work. By the work of Petersen and Tommasi [PT14, Pet13], the Gorenstein conjectures for the tautological 


\section{A tale of TWO TAUTOLOGICAL Rings}

rings of $\overline{\mathscr{M}}_{g, n}$ and $\mathscr{M}_{g, n}^{\text {ct }}$ are known to be false already when $g=2$. So it is tempting to believe that $\mathscr{R}\left(\mathscr{M}_{g}\right)$ may not be Gorenstein in general. Here we provide a possible explanation for this. Following Polishchuk's conjecture and philosophy in [Pol05], we may ask the following question.

Question. Are all relations of motivic nature?

This question, whose precise statement can be found in Conjecture 7.17, has a certain geometric (rather than numerical) flavor. A positive answer to it would contradict the Gorenstein conjecture, and thus give an alternative description of the tautological rings.

More recently, a new set of relations for various tautological rings (including $\mathscr{R}\left(\mathscr{C}_{g}^{[n]}\right)$ ) was conjectured by Pixton [Pix12] and proven by Pandharipande, Pixton and Zvonkine in cohomology [PPZ15], and by Janda in the Chow ring [Jan13]. In [Pix13, Appendix A], Pixton collected data about the discrepancies between his relations and the Gorenstein expectations for $\mathscr{R}\left(\mathscr{C}_{g}^{[n]}\right)$. Our third result then shows that after $g=24$ for $n=0, g=20$ for $n=1$, etc., the value $g=8$ is the ultimate critical value for any large $n$. It is one of the most interesting cases to test various Gorenstein properties.

Conventions. We work over an arbitrary field $k$. Throughout, Chow rings $\mathrm{CH}=\oplus_{i} \mathrm{CH}^{i}$ are with $\mathbb{Q}$-coefficients and graded by codimension. The symbol $\mathrm{CH}_{i}$ is used only relatively to a fixed base scheme $S$ : if $X$ is a smooth projective scheme over $S$ with connected fibers, we write $\mathrm{CH}_{i}(X)$ for its Chow group of relative dimension $i$ cycles with $\mathbb{Q}$-coefficients. We set $\mathfrak{s l}_{2}:=\mathbb{Q} \cdot e+\mathbb{Q} \cdot f+\mathbb{Q} \cdot h$, with $[e, f]=h,[h, e]=2 e$ and $[h, f]=-2 f$.

\section{Tautological rings around a relative curve}

We recall several tautological rings associated with a relative pointed curve. Then we focus on the universal situation, namely over the moduli space, where (an analog of) Faber's Gorenstein conjecture is stated. Throughout this section we work in the context of pointed curves, and we include a comparison with the unpointed counterpart at the end.

2.1. Let $k$ be a field, and let $S$ be a smooth connected variety of dimension $d$ over $k$. Consider a relative curve $p: C \rightarrow S$ of genus $g$, that is, a smooth projective scheme over $S$ with geometrically connected fibers of relative dimension 1 and of genus $g$. We assume $g>0$. We further assume that $C / S$ admits a section (marked point) $x_{0}: S \rightarrow C$.

For $n \geqslant 1$, denote by $p^{n}: C^{n} \rightarrow S$ (respectively, $p^{[n]}: C^{[n]} \rightarrow S$ ) the $n$th power (respectively, symmetric power) of $C$ relative to $S$. Write $\sigma_{n}: C^{n} \rightarrow C^{[n]}$ for the symmetrization map. For convenience we set $C^{0}=C^{[0]}:=S$.

2.2. We describe a few geometric classes in the Chow rings $\mathrm{CH}\left(C^{n}\right)$, which serve as the building blocks of the tautological rings. First, denote by $K \in \mathrm{CH}^{1}(C)$ the first Chern class of the relative cotangent bundle $\Omega_{C / S}^{1}$. Also write $\left[x_{0}\right]:=\left[x_{0}(S)\right] \in \mathrm{CH}^{1}(C)$.

Next, define classes

$$
\kappa_{i}:=p_{*}\left(K^{i+1}\right) \in \mathrm{CH}^{i}(S) \text { for } i \geqslant 0, \quad \psi:=x_{0}^{*}(K) \in \mathrm{CH}^{1}(S) .
$$

We have $\kappa_{0}=(2 g-2)[S]$, and it is convenient to write $\kappa_{-1}:=0$. Also note that $x_{0}^{*}\left(\left[x_{0}\right]\right)=-\psi$ by adjunction. 
Further, we view the $\mathrm{CH}\left(C^{n}\right)$ as $\mathrm{CH}(S)$-algebras by pulling back via $p^{n}$. We then keep the same notation $\left\{\kappa_{i}\right\}$ and $\psi$ for the pull-backs of these classes to $C^{n}$. For $1 \leqslant j \leqslant n$, let $\operatorname{pr}_{j}: C^{n} \rightarrow C$ be the projection to the $j$ th factor, and for $1 \leqslant k<l \leqslant n$, let $\operatorname{pr}_{k, l}: C^{n} \rightarrow C^{2}$ be the projection to the $k$ th and $l$ th factors. Denote by $\Delta$ the diagonal in $C^{2}$, and write

$$
\begin{aligned}
K_{j} & :=\operatorname{pr}_{j}^{*}(K) \in \mathrm{CH}^{1}\left(C^{n}\right), \\
{\left[x_{0, j}\right] } & :=\operatorname{pr}_{j}^{*}\left(\left[x_{0}\right]\right) \in \mathrm{CH}^{1}\left(C^{n}\right), \\
{\left[\Delta_{k, l}\right] } & :=\operatorname{pr}_{k, l}^{*}([\Delta]) \in \mathrm{CH}^{1}\left(C^{n}\right) .
\end{aligned}
$$

Definition 2.3. Let $n \geqslant 0$. The tautological ring of $C^{n}$, denoted by $\mathscr{R}\left(C^{n}\right)$, is the $\mathbb{Q}$-subalgebra of $\mathrm{CH}\left(C^{n}\right)$ generated by the classes $\left\{\kappa_{i}\right\}, \psi,\left\{K_{j}\right\}$ and $\left\{\left[x_{0, j}\right]\right\}$ (if $n \geqslant 1$ ), and $\left\{\left[\Delta_{k, l}\right]\right\}$ (if $n \geqslant 2$ ). Elements in $\mathscr{R}\left(C^{n}\right)$ are called tautological classes.

In particular, the ring $\mathscr{R}(S):=\mathscr{R}\left(C^{0}\right)$ is generated by $\left\{\kappa_{i}\right\}$ and $\psi$.

Remark 2.4. Alternatively, one may define $\mathscr{R}\left(C^{n}\right)$ in the style of Faber and Pandharipande [FP00, FP05]. For $n \geqslant 1$ and $m \geqslant 0$, consider maps

$$
T=\left(T_{1}, \ldots, T_{m}\right): C^{n} \rightarrow C^{m}
$$

such that each $T_{i}: C^{n} \rightarrow C$ is a projection of $C^{n}$ to one of its factors (when $m=0$ we set $T=p^{n}: C^{n} \rightarrow S$ ). These maps are called tautological maps. The tautological rings $\left\{\mathscr{R}\left(C^{n}\right)\right\}$ then form the smallest system of $\mathbb{Q}$-subalgebras satisfying $\left[x_{0}\right] \in \mathscr{R}(C)$ and stable under pullbacks and push-forwards via all tautological maps. The proof is not difficult and is essentially in [Loo95, Proposition 2.1].

2.5. For $n \geqslant 1$, the map $\sigma_{n}: C^{n} \rightarrow C^{[n]}$ induces an isomorphism of $\mathbb{Q}$-algebras

$$
\sigma_{n}^{*}: \mathrm{CH}\left(C^{[n]}\right) \stackrel{\sim}{\rightarrow} \mathrm{CH}\left(C^{n}\right)^{\mathfrak{S}_{n}},
$$

where $\mathrm{CH}\left(C^{n}\right)^{\mathfrak{S}_{n}}$ is the symmetric (or $\mathfrak{S}_{n}$-invariant) part of $\mathrm{CH}\left(C^{n}\right)$. Note that $\sigma_{n, *} \circ \sigma_{n}^{*}=n$ !. The tautological ring of $C^{[n]}$ is then defined to be

$$
\mathscr{R}\left(C^{[n]}\right):=\left(\sigma_{n}^{*}\right)^{-1}\left(\mathscr{R}\left(C^{n}\right)\right)=\frac{1}{n !} \sigma_{n, *}\left(\mathscr{R}\left(C^{n}\right)\right) .
$$

When $n=0$ we set $\mathscr{R}\left(C^{[0]}\right):=\mathscr{R}\left(C^{0}\right)(=\mathscr{R}(S))$.

2.6. Universal setting. The study of tautological rings was initiated by Mumford [Mum83], and later carried on extensively by Faber, Pandharipande, etc., in the context of various moduli spaces of curves and compactifications. We refer to [Fab99, Pan02, Fab13, FP13] for an overview of major questions.

Our situation concerns the moduli space of smooth pointed curves of genus $g$ over $k$, denoted by $\mathscr{M}_{g, 1}$ ( $g>0$ as before). It is isomorphic to the universal curve $\mathscr{C}_{g}$ over the moduli of smooth genus $g$ curves $\mathscr{M}_{g}$. We have $\operatorname{dim}\left(\mathscr{M}_{g, 1}\right)=\operatorname{dim}\left(\mathscr{M}_{g}\right)+1=3 g-3+1$. The stacky nature of $\mathscr{M}_{g, 1}$ does not play a role here. In fact, since $\mathscr{M}_{g, 1}$ admits a finite cover by a smooth connected variety (see [Mum83, Part I, Section 2]), its Chow theory with $\mathbb{Q}$-coefficients can be easily defined. In principle, one may regard $\mathscr{M}_{g, 1}$ as a smooth connected variety from here on.

Denote by $\mathscr{C}_{g, 1}$ the universal curve over $\mathscr{M}_{g, 1}$. The tautological rings $\mathscr{R}\left(\mathscr{C}_{g, 1}^{n}\right)$ and $\mathscr{R}\left(\mathscr{C}_{g, 1}^{[n]}\right)$ (and in particular $\mathscr{R}\left(\mathscr{M}_{g, 1}\right)$ ) are thus defined by the same recipe as above, with $S=\mathscr{M}_{g, 1}$. 


\section{A tale of TWO TAUTOLOGICAL Rings}

2.7. In [Fab99], Faber proposed the Gorenstein description of the tautological ring of $\mathscr{M}_{g}$ (see below). We adapt this idea to our context.

First, by the work of Looijenga [Loo95] and Faber [Fab97], we have

$$
\mathscr{R}^{i}\left(\mathscr{C}_{g, 1}^{n}\right)=0 \text { for } i>g-1+n \text { and } \mathscr{R}^{g-1+n}\left(\mathscr{C}_{g, 1}^{n}\right) \simeq \mathbb{Q} \text {. }
$$

Then for $0 \leqslant i \leqslant g-1+n$, consider the pairing

$$
\mathscr{R}^{i}\left(\mathscr{C}_{g, 1}^{n}\right) \times \mathscr{R}^{g-1+n-i}\left(\mathscr{C}_{g, 1}^{n}\right) \dot{\rightarrow} \mathscr{R}^{g-1+n}\left(\mathscr{C}_{g, 1}^{n}\right) \simeq \mathbb{Q} .
$$

The following is an analog of Faber's Gorenstein conjecture.

SpeCulation 2.8. For $n \geqslant 0$ and $0 \leqslant i \leqslant g-1+n$, the pairing (2.2) is perfect. In other words, the ring $\mathscr{R}\left(\mathscr{C}_{g, 1}^{n}\right)$ is Gorenstein with socle in degree $g-1+n$.

Note that $\mathscr{R}^{g-1+n}\left(\mathscr{C}_{g, 1}^{n}\right)$ is $\mathfrak{S}_{n}$-invariant. Restricting to the symmetric part, one would have the same Gorenstein property for $\mathscr{R}\left(\mathscr{C}_{g, 1}^{[n]}\right)$; that is, there is a perfect pairing

$$
\mathscr{R}^{i}\left(\mathscr{C}_{g, 1}^{[n]}\right) \times \mathscr{R}^{g-1+n-i}\left(\mathscr{C}_{g, 1}^{[n]}\right) \rightarrow \mathscr{R}^{g-1+n}\left(\mathscr{C}_{g, 1}^{[n]}\right) \simeq \mathbb{Q}
$$

2.9. Speculation 2.8 is closely related to the Gorenstein conjecture for the tautological ring of $\mathscr{M}_{g, n}^{\mathrm{rt}}$, the moduli space of stable $n$-pointed genus $g$ curves with rational tails. The latter is stated in [Pan02]. In fact, for $n=0$ we have exactly $\mathscr{C}_{g, 1}^{0}=\mathscr{M}_{g, 1}=\mathscr{M}_{g, 1}^{\mathrm{rt}}$. More generally there is a surjective map $\mathscr{M}_{g, n+1}^{\mathrm{rt}} \rightarrow \mathscr{C}_{g, 1}^{n}$, which can be expressed as a sequence of blow-ups. The Gorenstein properties on both sides were recently shown to be equivalent by Petersen [Pet15].

Moreover, Speculation 2.8 was proven for $g=1,2$ by Tavakol [Tav11, Tav14] (he went on to prove the Gorenstein conjecture for the tautological rings of $\mathscr{M}_{1, n}^{\mathrm{rt}}$ and $\mathscr{M}_{2, n}^{\mathrm{rt}}$ ).

2.10. Unpointed version. Having stated everything in terms of pointed curves, we provide a translation to the classical, unpointed version.

Consider the universal curve $\mathscr{C}_{g}$ over $\mathscr{M}_{g}$ and its relative powers $\mathscr{C}_{g}^{n}$ and symmetric powers $\mathscr{C}_{g}^{[n]}$. The tautological rings $\mathscr{R}\left(\mathscr{C}_{g}^{n}\right)$ are defined to be generated by $\left\{\kappa_{i}\right\},\left\{K_{j}\right\}$ and $\left\{\left[\Delta_{k, l}\right]\right\}$, that is, classes not involving the section $x_{0}$. One also defines $\mathscr{R}\left(\mathscr{M}_{g}\right)$ and $\mathscr{R}\left(\mathscr{C}_{g}^{[n]}\right)$ accordingly.

Since $\mathscr{M}_{g, 1} \simeq \mathscr{C}_{g}$, there are isomorphisms

$$
\mathscr{C}_{g, 1}^{n} \simeq \mathscr{C}_{g}^{n} \times \mathscr{M}_{g} \mathscr{M}_{g, 1} \simeq \mathscr{C}_{g}^{n+1} \quad \text { and } \quad \mathscr{C}_{g, 1}^{[n]} \simeq \mathscr{C}_{g}^{[n]} \times \mathscr{M}_{g} \mathscr{M}_{g, 1} \simeq \mathscr{C}_{g}^{[n]} \times \mathscr{M}_{g} \mathscr{C}_{g} .
$$

Under these isomorphisms, we have a dictionary for tautological classes: the class $\psi \in \mathscr{R}\left(\mathscr{C}_{g, 1}^{n}\right)$ corresponds to $K_{n+1} \in \mathscr{R}\left(\mathscr{C}_{g}^{n+1}\right)$, and $\left[x_{0, j}\right] \in \mathscr{R}\left(\mathscr{C}_{g, 1}^{n}\right)$ corresponds to $\left[\Delta_{j, n+1}\right] \in \mathscr{R}\left(\mathscr{C}_{g}^{n+1}\right)$. The dictionary gives isomorphisms of $\mathbb{Q}$-algebras

$$
\mathscr{R}\left(\mathscr{C}_{g, 1}^{n}\right) \simeq \mathscr{R}\left(\mathscr{C}_{g}^{n+1}\right) \quad \text { and } \quad \mathscr{R}\left(\mathscr{C}_{g, 1}^{[n]}\right) \simeq \mathscr{R}\left(\mathscr{C}_{g}^{n+1}\right)^{\mathfrak{S}_{n}}
$$

where $\mathfrak{S}_{n}$ acts on the first $n$ factors of $\mathscr{C}_{g}^{n+1}$.

One can also formulate the corresponding Gorenstein properties for $\mathscr{R}\left(\mathscr{C}_{g}^{n}\right)$ and $\mathscr{R}\left(\mathscr{C}_{g}^{[n]}\right)$ (note that $\mathscr{R}^{g-2+n}\left(\mathscr{C}_{g}^{n}\right)=\mathscr{R}^{g-2+n}\left(\mathscr{C}_{g}^{[n]}\right) \simeq \mathbb{Q}$ ). It is immediate that the Gorenstein properties for $\mathscr{R}\left(\mathscr{C}_{g, 1}^{n}\right)$ and $\mathscr{R}\left(\mathscr{C}_{g}^{n+1}\right)$ are equivalent, and that $\mathscr{R}\left(\mathscr{C}_{g, 1}^{[n]}\right)$ being Gorenstein implies $\mathscr{R}\left(\mathscr{C}_{g}^{[n+1]}\right)$ being Gorenstein. 


\section{The Chow ring of the relative Jacobian}

We review three important structures on the Chow ring of the relative Jacobian, namely the Beauville decomposition, the Fourier transform and the Lefschetz decomposition. We also discuss their motivic background. A picture called the Dutch house is presented to visualize these structures.

3.1. As in the previous section, we take $S$ a smooth connected variety of dimension $d$ over $k$. Let $p: C \rightarrow S$ be a relative curve of genus $g>0$, together with a section $x_{0}: S \rightarrow C$. Denote by $\pi: J \rightarrow S$ the associated relative Jacobian. By definition $J:=\operatorname{Pic}^{0}(C / S)$ is an abelian scheme of relative dimension $g$. The section $x_{0}$ induces an embedding $\iota: C \hookrightarrow J$, which sends (locally) a section $x$ of $C / S$ to $\iota(x):=\mathscr{O}_{C}\left(x-x_{0}\right)$. The composition $\iota \circ x_{0}$ is then the zero section $o: S \rightarrow J$. To summarize, we have the following diagram:

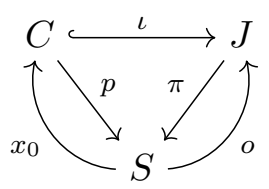

3.2. The (abelian) group structure on $J$ gives the addition and scalar multiplication maps

$$
\mu: J \times{ }_{S} J \rightarrow J \text { and }[N]: J \rightarrow J \text { for } N \in \mathbb{Z} .
$$

The Chow ring $\mathrm{CH}(J)$ carries a second ring structure called the Pontryagin product, denoted by $*$. It sends $\alpha \in \mathrm{CH}^{i}(J)$ and $\beta \in \mathrm{CH}^{j}(J)$ to

$$
\alpha * \beta:=\mu_{*}\left(\operatorname{pr}_{1}^{*}(\alpha) \cdot \operatorname{pr}_{2}^{*}(\beta)\right) \in \mathrm{CH}^{i+j-g}(J),
$$

where $\mathrm{pr}_{1}, \mathrm{pr}_{2}: J \times_{S} J \rightarrow J$ are the two projections.

3.3. Beauville decomposition. By the work of Beauville [Bea86] (later generalized to the relative setting by Deninger and Murre [DM91]), the Chow ring $\mathrm{CH}(J)$ can be decomposed into eigenspaces with respect to the action of $[N]$. More precisely, for $0 \leqslant i \leqslant g+d$, we have

$$
\mathrm{CH}^{i}(J)=\bigoplus_{j=\max \{i-g, 2 i-2 g\}}^{\min \{i+d, 2 i\}} \mathrm{CH}_{(j)}^{i}(J),
$$

with

$$
\mathrm{CH}_{(j)}^{i}(J):=\left\{\alpha \in \mathrm{CH}^{i}(J):[N]^{*}(\alpha)=N^{2 i-j} \alpha \text { for all } N \in \mathbb{Z}\right\} .
$$

The decomposition is multiplicative: for $\alpha \in \mathrm{CH}_{(j)}^{i}(J)$ and $\beta \in \mathrm{CH}_{(l)}^{k}(J)$, we have $\alpha \cdot \beta \in$ $\mathrm{CH}_{(j+l)}^{i+k}(J)$ and $\alpha * \beta \in \mathrm{CH}_{(j+l)}^{i+k-g}(J)$.

3.4. To introduce the two other structures, recall that $J$ has a canonical principal polarization $\lambda: J \stackrel{\sim}{\rightarrow} J^{t}$. Denote by $\mathscr{P}$ (the pull-back of) the Poincaré line bundle on $J \times_{S} J$, trivialized along the two zero sections. We write $\ell \in \mathrm{CH}^{1}\left(J \times_{S} J\right)$ for its first Chern class.

Also associated with the polarization is a relatively ample divisor class $\theta \in \mathrm{CH}_{(0)}^{1}(J)$. There are identities

$$
\theta=-\frac{1}{2} \Delta^{*}(\ell) \quad \text { and } \quad \ell=\operatorname{pr}_{1}^{*}(\theta)+\operatorname{pr}_{2}^{*}(\theta)-\mu^{*}(\theta)
$$




\section{A tale of TWO TAUTOLOGICAL Rings}

where $\Delta: J \rightarrow J \times{ }_{S} J$ is the diagonal map. Note that we adopted the sign convention explained in [Bea10, Section 1.6].

3.5. Fourier transform. The class $\exp (\ell)$, viewed as a correspondence, induces the Fourier transform $\mathscr{F}: \mathrm{CH}(J) \rightarrow \mathrm{CH}(J)$. It sends $\alpha \in \mathrm{CH}(J)$ to

$$
\mathscr{F}(\alpha):=\operatorname{pr}_{2, *}\left(\operatorname{pr}_{1}^{*}(\alpha) \cdot \exp (\ell)\right) .
$$

We have the following identities (see [Bea86, Section 1] or [DM91, Section 2]):

$$
\mathscr{F}(\alpha * \beta)=\mathscr{F}(\alpha) \cdot \mathscr{F}(\beta), \quad \mathscr{F}(\alpha \cdot \beta)=(-1)^{g} \mathscr{F}(\alpha) * \mathscr{F}(\beta) \quad \text { and } \quad \mathscr{F} \circ \mathscr{F}=(-1)^{g}[-1]^{*} .
$$

Further, there are isomorphisms

$$
\mathscr{F}: \mathrm{CH}_{(j)}^{i}(J) \stackrel{\sim}{\rightarrow} \mathrm{CH}_{(j)}^{g-i+j}(J) .
$$

Hence, by applying $\mathscr{F}$ to $\mathrm{CH}^{i}(J)$ and by collecting components of different codimensions, one recovers the Beauville decomposition.

3.6. Lefschetz decomposition. By Künnemann [Kün93], the classical Lefschetz decomposition in cohomology can be lifted to the Chow ring of an abelian scheme. In the case of the relative Jacobian, Polishchuk [Pol07a] showed that the decomposition can be reconstructed geometrically.

Write $[C]:=[\iota(C)] \in \mathrm{CH}^{g-1}(J)$ for the curve class, and $[C]_{(j)} \in \mathrm{CH}_{(j)}^{g-1}(J)$ for the components of $[C]$ in the Beauville decomposition. First, we have identities

$$
\theta=\frac{1}{(g-1) !}[C]_{(0)}^{*(g-1)}=-\mathscr{F}\left([C]_{(0)}\right) .
$$

On $\mathrm{CH}(J)$, define operators

$$
\begin{aligned}
& e: \mathrm{CH}_{(j)}^{i}(J) \rightarrow \mathrm{CH}_{(j)}^{i+1}(J), \quad \alpha \mapsto-\theta \cdot \alpha, \\
& f: \mathrm{CH}_{(j)}^{i}(J) \rightarrow \mathrm{CH}_{(j)}^{i-1}(J), \quad \alpha \mapsto-[C]_{(0)} * \alpha, \\
& h: \mathrm{CH}_{(j)}^{i}(J) \rightarrow \mathrm{CH}_{(j)}^{i}(J), \quad \alpha \mapsto(2 i-j-g) \alpha .
\end{aligned}
$$

Then the operators $e, f$ and $h$ generate a $\mathbb{Q}$-linear representation of $\mathfrak{s l}_{2}$, which induces the Lefschetz decomposition on $\mathrm{CH}(J)$. See [Pol07a, Theorem 2.6] for the proof of this statement. Note that we followed Polishchuk's sign convention by setting $e$ to be $-\theta$.

From now on we refer to (3.6) as the $\mathfrak{s l}_{2}$-action.

Remark 3.7. There is an identity (see [Bea04, Section 2.3(iv)] or [Pol08, Lemma 1.4])

$$
\mathscr{F}=\exp (e) \exp (-f) \exp (e) \quad \text { on } \mathrm{CH}(J) .
$$

This means that if we represent $e, f$ by the matrices $\left(\begin{array}{ll}0 & 1 \\ 0 & 0\end{array}\right),\left(\begin{array}{ll}0 & 0 \\ 1 & 0\end{array}\right) \in \mathfrak{s l}_{2}(\mathbb{Q})$, respectively, then $\mathscr{F}$ corresponds to the matrix $\left(\begin{array}{cc}0 & 1 \\ -1 & 0\end{array}\right) \in \mathrm{SL}_{2}(\mathbb{Q})$. As the Fourier transform induces the Beauville decomposition, we see that among the three structures given above, the Lefschetz decomposition (or the $\mathfrak{s l}_{2}$-action) may be viewed as the deepest.

3.8. Motivic background. We briefly discuss the motivic interpretation of the structures above. See [DM91] and [Kün93] for more details. 
Q. Y IN

Denote by $R(J / S)$ the relative Chow motive of $J$. Then $R(J / S)$ admits a decomposition

$$
R(J / S)=\bigoplus_{i=0}^{2 g} R^{i}(J / S),
$$

with $[N]^{*}$ acting on $R^{i}(J / S)$ by multiplication by $N^{i}$. It follows that

$$
\mathrm{CH}_{(j)}^{i}(J)=\mathrm{CH}^{i}\left(R^{2 i-j}(J / S)\right) \text {. }
$$

Moreover, the Fourier transform $\mathscr{F}$ induces isomorphisms

$$
\mathscr{F}: R^{i}(J / S) \stackrel{\sim}{\rightarrow} R^{2 g-i}(J / S)(g-i),
$$

where (-) stands for Tate twists. Finally, we have the motivic Lefschetz decomposition

$$
R^{i}(J / S)=\bigoplus_{k=\max \{0, i-g\}}^{\lfloor i / 2\rfloor} e^{k}\left(R_{\operatorname{prim}}^{i-2 k}(J / S)(-k)\right),
$$

where $R_{\text {prim }}^{i}(J / S)$ is the primitive part of $R^{i}(J / S)$ with respect to the $\mathfrak{s l}_{2}$-action.

3.9. We find it convenient to replace the codimension grading on $\mathrm{CH}(J)$ by a new, motivic grading. We write

$$
\mathrm{CH}_{(i, j)}(J):=\mathrm{CH}_{(j)}^{(i+j) / 2}(J), \quad \text { or equivalently } \quad \mathrm{CH}_{(2 i-j, j)}(J):=\mathrm{CH}_{(j)}^{i}(J),
$$

so that $[N]^{*}$ acts on $\mathrm{CH}_{(i, j)}(J)$ by multiplication by $N^{i}$. In other words, we set

$$
\mathrm{CH}_{(i, j)}(J)=\mathrm{CH}\left(R^{i}(J / S)\right) \cap \mathrm{CH}_{(j)}(J) .
$$

The Beauville decomposition (3.3) then takes on the form

$$
\mathrm{CH}(J)=\bigoplus_{i, j} \mathrm{CH}_{(i, j)}(J)
$$

with $0 \leqslant i \leqslant 2 g, \max \{-i, i-2 g\} \leqslant j \leqslant \min \{i, 2 g-i\}+2 d$ and $i+j$ even.

Expressions under the new grading are simple (if not simpler): for $\alpha \in \mathrm{CH}_{(i, j)}(J)$ and $\beta \in$ $\mathrm{CH}_{(k, l)}(J)$, we have $\alpha \cdot \beta \in \mathrm{CH}_{(i+k, j+l)}(J)$ and $\alpha * \beta \in \mathrm{CH}_{(i+k-2 g, j+l)}(J)$. Also, the Fourier transform $\mathscr{F}$ induces

$$
\mathscr{F}: \mathrm{CH}_{(i, j)}(J) \stackrel{\sim}{\rightarrow} \mathrm{CH}_{(2 g-i, j)}(J) .
$$

Further, we have $\theta \in \mathrm{CH}_{(2,0)}(J)$. The $\mathfrak{s l}_{2}$-action in (3.6) becomes

$$
\begin{array}{ll}
e: \mathrm{CH}_{(i, j)}(J) \rightarrow \mathrm{CH}_{(i+2, j)}(J), & \alpha \mapsto-\theta \cdot \alpha, \\
f: \mathrm{CH}_{(i, j)}(J) \rightarrow \mathrm{CH}_{(i-2, j)}(J), & \alpha \mapsto-[C]_{(0)} * \alpha, \\
h: \mathrm{CH}_{(i, j)}(J) \rightarrow \mathrm{CH}_{(i, j)}(J), & \alpha \mapsto(i-g) \alpha .
\end{array}
$$

3.10. Dutch house. We present a useful picture that illustrates the structures given above and combines the motivic aspect. It also allows us to make clear statements without complicated indices. The picture is inspired by [Moo09, Figure 1]. We call it the Dutch house due to its resemblance to a traditional Dutch trapgevel (crow-stepped gable).

In Figure 1, the $(i, j)$ th block represents the component $\mathrm{CH}_{(i, j)}(J)$ in the Beauville decomposition. The columns then read the motivic decomposition $R(J / S)=\oplus_{i} R^{i}(J / S)$, and the rows 
A tale of TWO TAUtological Rings

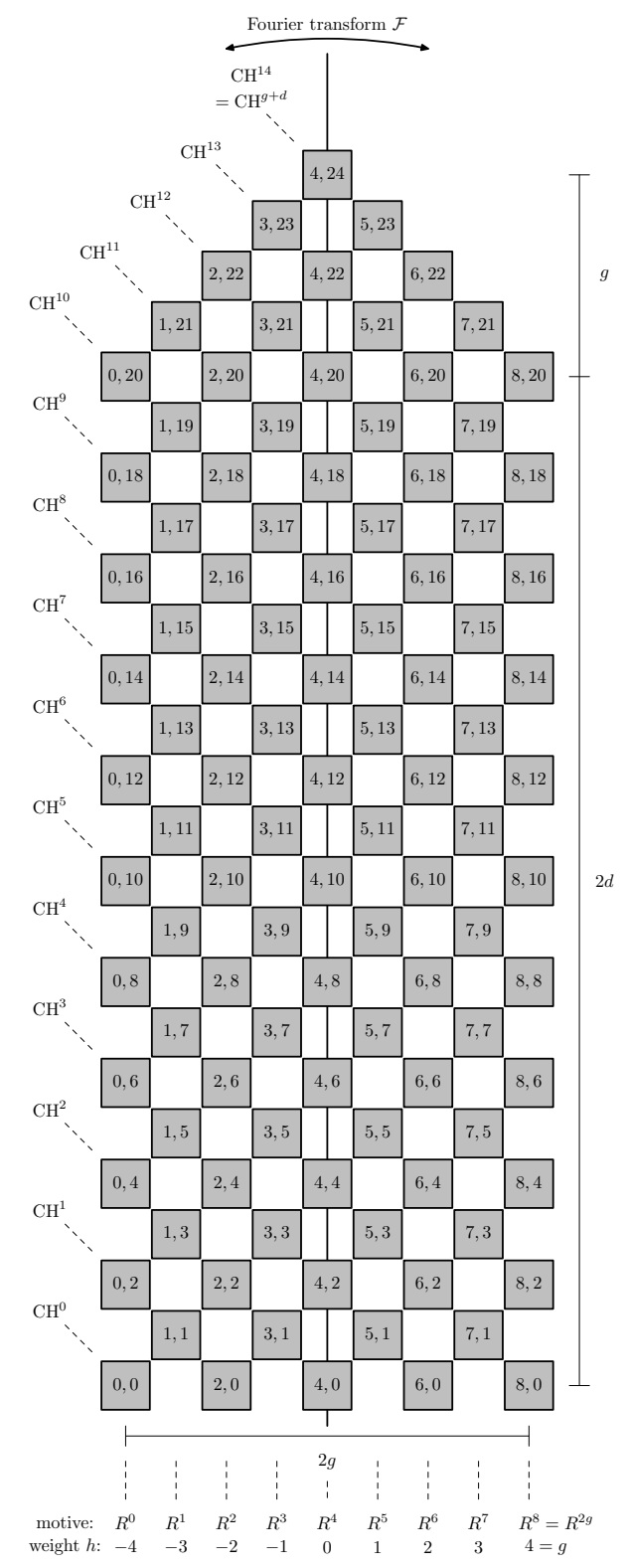

Figure 1. Mon dessin $n^{\circ} 1$ : the outside of the Dutch house $(g=4$ and $d=10)$

read Beauville's grading $j$. As a result, components with the same codimension lie on a dashed line from upper left to lower right.

It is not difficult to verify that the house shape results from the precise index range of (3.7). The width of the house depends on the genus $g$, while the height (without roof) depends on $d=$ $\operatorname{dim}(S)$. In particular, when $S=k$, the house reduces to the roof only. Here Figure 1 is drawn based on the universal Jacobian over $S=\mathscr{M}_{4,1}$, with $d=\operatorname{dim}\left(\mathscr{M}_{4,1}\right)=10$.

As is shown in the picture, the Fourier transform $\mathscr{F}$ acts as the reflection over the middle vertical line. The operator $e$ in the $\mathfrak{s l}_{2}$-action shifts classes to the right by 2 blocks, while $f$ shifts classes to the left by 2 blocks. Finally, the middle column of the house has weight zero with respect to $h$. 
Remark 3.11. Note that we have not drawn the components $\mathrm{CH}_{(i, j)}(J)$ with negative $j$. On the one hand, when $S=k$ the Beauville conjecture predicts the vanishing of those components (see [Bea86, Section 2]). On the other hand, all classes we shall encounter are in $\mathrm{CH}_{(i, j)}(J)$ with $j \geqslant 0$, that is, inside the house.

3.12. The isomorphisms of motives (see [DM91, Example 1.4])

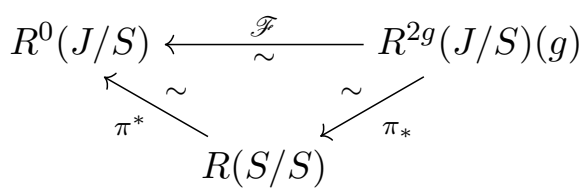

induce the following isomorphisms of $\mathbb{Q}$-algebras:

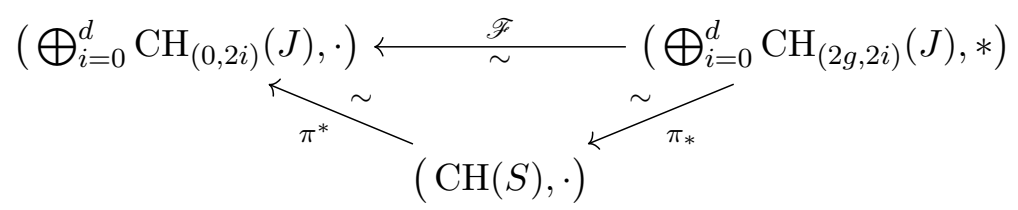

The gradings are preserved as $\pi^{*}: \mathrm{CH}^{i}(S) \stackrel{\sim}{\rightarrow} \mathrm{CH}_{(0,2 i)}(J)$ and $\pi_{*}: \mathrm{CH}_{(2 g, 2 i)}(J) \stackrel{\sim}{\rightarrow} \mathrm{CH}^{i}(S)$.

In particular, the Chow ring $\mathrm{CH}(S)$ may be regarded as a $\mathbb{Q}$-subalgebra of $(\mathrm{CH}(J), \cdot)$ via $\pi^{*}$, or as a $\mathbb{Q}$-subalgebra of $(\mathrm{CH}(J), *)$ via $\pi_{*}$. In terms of the Dutch house, we may identify $\mathrm{CH}(S)$ with the zeroth column or with the $2 g$ th column of the house.

\section{The tautological ring of the relative Jacobian}

We define the tautological ring $\mathscr{T}(J)$ of the relative Jacobian. We determine its generators and give explicit formulae for the $\mathfrak{s l}_{2}$-action in terms of the generators. A consequence is that by pulling back via $\pi^{*}: \mathrm{CH}(S) \rightarrow \mathrm{CH}(J)$, one can identify $\mathscr{R}(S)$ with the $\mathbb{Q}$-subalgebra of $\mathscr{T}(J)$ located on the zeroth column of the Dutch house. Throughout this section we work in the setting of (3.1).

DeFinition 4.1. The tautological ring of $J$, denoted by $\mathscr{T}(J)$, is the smallest $\mathbb{Q}$-subalgebra of $(\mathrm{CH}(J), \cdot, *)$ (that is, subalgebra with respect to both $\cdot$ and $*$ ) that contains $[C] \in \mathrm{CH}^{g-1}(J)$ and that is stable under $[N]^{*}$ for all $N \in \mathbb{Z}$. Elements in $\mathscr{T}(J)$ are called tautological classes.

This notion of tautological ring was introduced by Beauville [Bea04] in the context of a Jacobian variety and modulo algebraic equivalence. Since then it has been studied in various contexts. We refer to [Pol05, Pol07b, Her07, vdGK07, Moo09] for more details. In the relative setting, Polishchuk considered a much bigger tautological ring, including all classes in $\pi^{*}(\mathrm{CH}(S))$ (see [Pol07a, Section 4]). Our minimalist version turns out to be the right one for studying the tautological ring of $S$.

Remark 4.2. (i) The ring $\mathscr{T}(J)$ is stable under the Beauville decomposition, the Fourier transform and the Lefschetz decomposition (or the $\mathfrak{s l}_{2}$-action). Indeed, by definition $\mathscr{T}(J)$ is graded by codimension. Also, by applying $[N]^{*}$ with various $N$ we have $[C]_{(0)} \in \mathscr{T}(J)$. Then it follows from (3.5) and (3.6) that $\mathscr{T}(J)$ is stable under the $\mathfrak{s l}_{2}$-action. As is shown in Remark 3.7, this implies the stability of $\mathscr{T}(J)$ under all three structures on $\mathrm{CH}(J)$. 


\section{A tale of TWO TAUTOLOGiCAL RiNGS}

(ii) Alternatively, one may define $\mathscr{T}(J)$ to be the smallest $\mathbb{Q}$-subalgebra of $(\mathrm{CH}(J), \cdot)$ containing $[C] \in \mathrm{CH}^{g-1}(J)$ and stable under the $\mathfrak{s l}_{2}$-action (and thus stable under the other two structures). In fact, being stable under the Beauville decomposition is the same as being stable under $[N]^{*}$. Since $\mathscr{F}$ interchanges and $*$, the ring is also stable under $*$.

4.3. Since the two products - and $*$ do not commute with each other, it is a priori not clear whether $\mathscr{T}(J)$ is finitely generated. We give an affirmative answer to this question by writing down an explicit set of generators.

Recall that $[C]_{(j)} \in \mathscr{T}_{(2 g-2-j, j)}(J)$. By the index range of $(3.7)$, we have $[C]_{(j)}=0$ for $j<0$ and for $j>2 g-2$. Now consider for $i \leqslant j+2$ and $i+j$ even

$$
\theta^{(j-i+2) / 2} \cdot[C]_{(j)} \in \mathscr{T}_{(2 g-i, j)}(J) .
$$

Denote its Fourier dual by

$$
p_{i, j}:=\mathscr{F}\left(\theta^{(j-i+2) / 2} \cdot[C]_{(j)}\right) \in \mathscr{T}_{(i, j)}(J) .
$$

As examples we have $p_{2,0}=\mathscr{F}\left([C]_{(0)}\right)=-\theta$ and $p_{0,0}=\mathscr{F}\left(\theta \cdot[C]_{(0)}\right)=g[J]$. The index range of (3.7) implies $p_{i, j}=0$ when $i<0$ or $j<0$ or $j>2 g-2$.

Figure 2 depicts the classes $\left\{p_{i, j}\right\}$ inside the Dutch house with $g=8$. Also shown in the picture is the pull-back of the class $\psi$ via $\pi^{*}$, again denoted by $\psi$, which lies in $\mathrm{CH}_{(0,2)}(J)$. Note that when $d=\operatorname{dim}(S)$ is small, classes above the roof vanish as well.

4.4. By definition, the operator $e$ in the $\mathfrak{s l}_{2}$-action is the intersection with $p_{2,0}$. Also, it is not difficult to verify that

$$
f\left(p_{i, j}\right)=p_{i-2, j} \cdot
$$

One of the questions is to calculate the class $f\left(p_{i, j} p_{k, l}\right)$. This turns out to be the key to the following theorem.

THEOREM 4.5. (i) The ring $\mathscr{T}(J)$ coincides with the $\mathbb{Q}$-subalgebra of $(\mathrm{CH}(J), \cdot)$ generated by the classes $\left\{p_{i, j}\right\}$ and $\psi$. In particular, it is finitely generated.

(ii) The operator $f$ acts on polynomials in $\left\{p_{i, j}\right\}$ and $\psi$ via the degree 2 differential operator

$$
\mathscr{D}:=\frac{1}{2} \sum_{i, j, k, l}\left(\psi p_{i-1, j-1} p_{k-1, l-1}-\left(\begin{array}{c}
i+k-2 \\
i-1
\end{array}\right) p_{i+k-2, j+l}\right) \partial p_{i, j} \partial p_{k, l}+\sum_{i, j} p_{i-2, j} \partial p_{i, j}
$$

To prove the theorem, we begin with a lemma that computes the pull-back of $\theta$ via the embedding $\iota: C \hookrightarrow J$. It is probably known to experts, and is implicit in [Pol07a, Theorem 2.6].

LEMMA 4.6. We have the identity

$$
\iota^{*}(\theta)=\frac{1}{2} K+\left[x_{0}\right]+\frac{1}{2} \psi \quad \text { in } \mathrm{CH}^{1}(C) .
$$

Proof. The goal is to calculate $\iota^{*}(\theta)=-\iota^{*}\left(\mathscr{F}\left([C]_{(0)}\right)\right)$ and we start from $\iota^{*}(\mathscr{F}([C]))$. Consider 


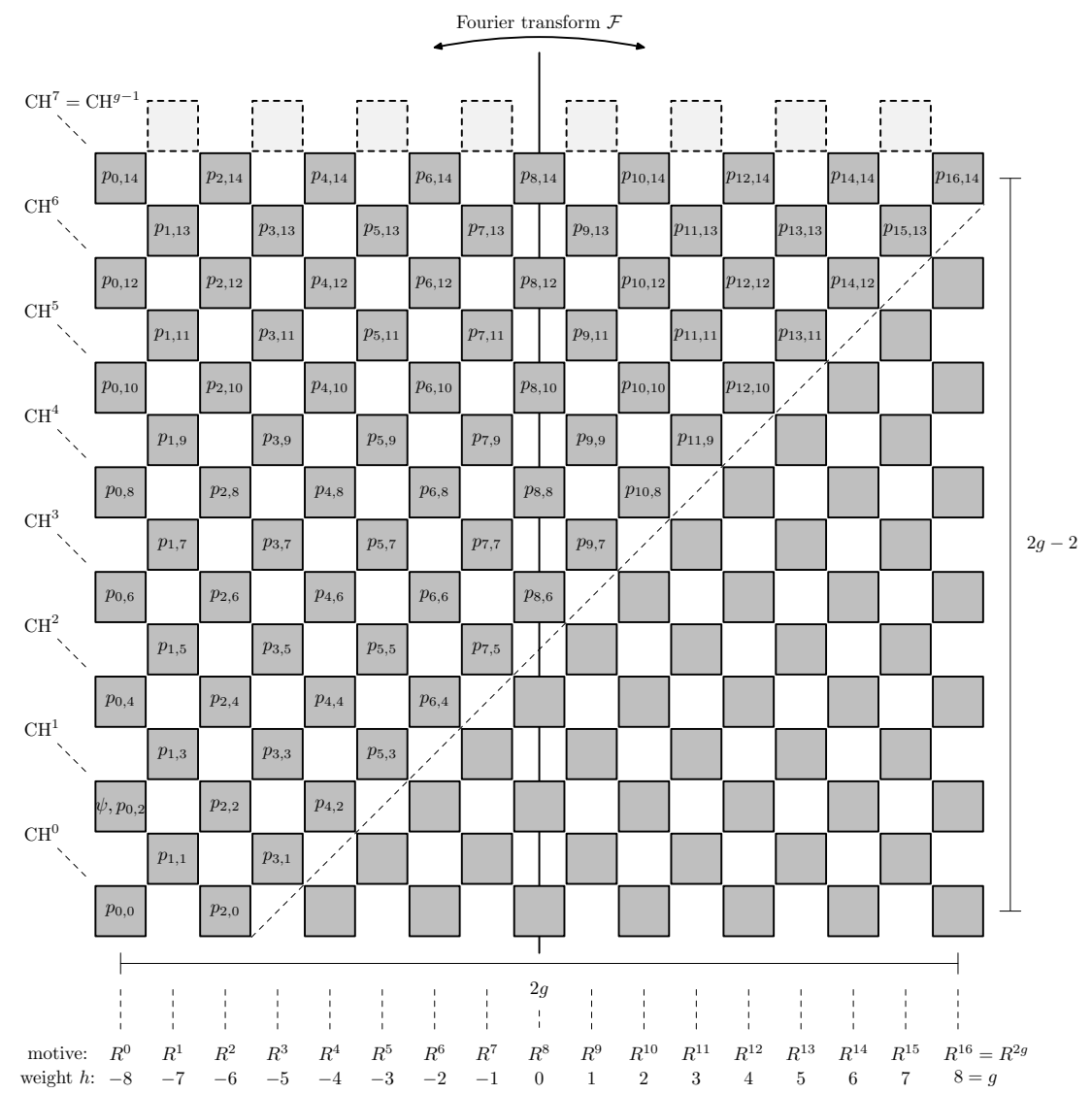

Figure 2. Mon dessin $n^{\circ} 2$ : the inside of the Dutch house $(g=8)$

the following three cartesian squares:

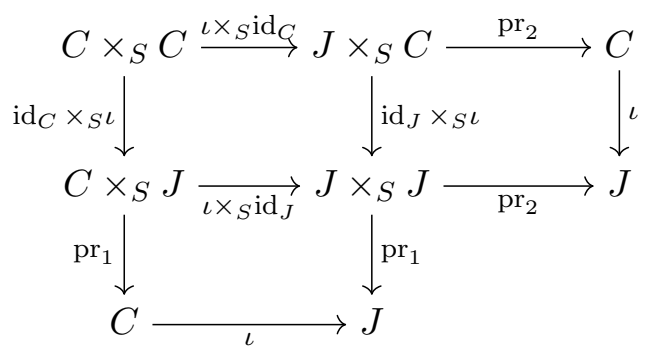

where $\mathrm{pr}_{1}$ and $\mathrm{pr}_{2}$ stand for the two projections in all cases. Then we have

$$
\begin{aligned}
\iota^{*}(\mathscr{F}([C])) & =\iota^{*} \operatorname{pr}_{2, *}\left(\operatorname{pr}_{1}^{*} \iota_{*}([C]) \cdot \exp (\ell)\right) \\
& =\operatorname{pr}_{2, *}\left(\operatorname{id}_{J} \times_{S} \iota\right)^{*}\left(\left(\iota \times_{S} \operatorname{id}_{J}\right)_{*} \operatorname{pr}_{1}^{*}([C]) \cdot \exp (\ell)\right) \\
& =\operatorname{pr}_{2, *}\left(\operatorname{id}_{J} \times_{S} \iota\right)^{*}\left(\left(\iota \times_{S} \operatorname{id}_{J}\right)_{*}\left(\left[C \times_{S} J\right]\right) \cdot \exp (\ell)\right) \\
& =\operatorname{pr}_{2, *}\left(\operatorname{id}_{J} \times_{S} \iota\right)^{*}\left(\iota \times_{S} \operatorname{id}_{J}\right)_{*}\left(\iota \times_{S} \operatorname{id}_{J}\right)^{*}(\exp (\ell)) \\
& =\operatorname{pr}_{2, *}\left(\iota \times_{S} \operatorname{id}_{C}\right)_{*}\left(\operatorname{id}_{C} \times_{S} \iota\right)^{*}\left(\iota \times_{S} \operatorname{id}_{J}\right)^{*}(\exp (\ell)) \\
& =\operatorname{pr}_{2, *}\left(\iota \times_{S} \iota\right)^{*}(\exp (\ell)) \\
& =\operatorname{pr}_{2, *}\left(\exp \left(\left(\iota \times_{S} \iota\right)^{*}(\ell)\right)\right) .
\end{aligned}
$$




\section{A tale of TWO TAUTOLOGICAL Rings}

The second identity in (3.4) and the theorem of the square imply (see [Pol07a, Formula (2.1)])

$$
\left(\iota \times_{S} \iota\right)^{*}(\ell)=[\Delta]-\operatorname{pr}_{1}^{*}\left(\left[x_{0}\right]\right)-\operatorname{pr}_{2}^{*}\left(\left[x_{0}\right]\right)-\psi,
$$

where $\Delta$ is the diagonal in $C \times{ }_{S} C$. It follows that

$$
\begin{aligned}
\iota^{*}(\mathscr{F}([C])) & =\operatorname{pr}_{2, *}\left(\exp \left([\Delta]-\operatorname{pr}_{1}^{*}\left(\left[x_{0}\right]\right)-\operatorname{pr}_{2}^{*}\left(\left[x_{0}\right]\right)-\psi\right)\right) \\
& =\operatorname{pr}_{2, *}\left(\exp \left([\Delta]-\operatorname{pr}_{1}^{*}\left(\left[x_{0}\right]\right)\right)\right) \cdot \exp \left(-\left[x_{0}\right]-\psi\right) .
\end{aligned}
$$

Observe that on the left-hand side of (4.4), we have

$$
\iota^{*}(\mathscr{F}([C]))=\sum_{j=0}^{2 g-2} \iota^{*}\left(\mathscr{F}\left([C]_{(j)}\right)\right),
$$

with $\iota^{*}\left(\mathscr{F}\left([C]_{(j)}\right)\right) \in \mathrm{CH}^{j+1}(C)$. Hence $\iota^{*}\left(\mathscr{F}\left([C]_{(0)}\right)\right)$ is just the codimension 1 component of $\iota^{*}(\mathscr{F}([C]))$. Expanding the exponentials in (4.4) while keeping track of the codimension, we obtain

$$
\begin{aligned}
\iota^{*}\left(\mathscr{F}\left([C]_{(0)}\right)\right) & =\operatorname{pr}_{2, *}\left(\frac{1}{2}\left([\Delta]-\operatorname{pr}_{1}^{*}\left(\left[x_{0}\right]\right)\right)^{2}\right)-\operatorname{pr}_{2, *}\left([\Delta]-\operatorname{pr}_{1}^{*}\left(\left[x_{0}\right]\right)\right) \cdot\left(\left[x_{0}\right]+\psi\right) \\
& =\operatorname{pr}_{2, *}\left(\frac{1}{2}\left([\Delta]-\operatorname{pr}_{1}^{*}\left(\left[x_{0}\right]\right)\right)^{2}\right) \\
& =\frac{1}{2} \operatorname{pr}_{2, *}([\Delta] \cdot[\Delta])-\operatorname{pr}_{2, *}\left([\Delta] \cdot \operatorname{pr}_{1}^{*}\left(\left[x_{0}\right]\right)\right)+\frac{1}{2} \operatorname{pr}_{2, *} \operatorname{pr}_{1}^{*}\left(\left[x_{0}\right] \cdot\left[x_{0}\right]\right)
\end{aligned}
$$

The first two terms in the previous expression are easily calculated: we have

$$
\operatorname{pr}_{2, *}([\Delta] \cdot[\Delta])=-K \text { and } \operatorname{pr}_{2, *}\left([\Delta] \cdot \operatorname{pr}_{1}^{*}\left(\left[x_{0}\right]\right)\right)=\left[x_{0}\right]
$$

For the term $\operatorname{pr}_{2, *} \operatorname{pr}_{1}^{*}\left(\left[x_{0}\right] \cdot\left[x_{0}\right]\right)$, consider the following cartesian square:

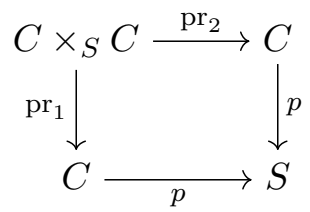

Then we have

$$
\operatorname{pr}_{2, *} \operatorname{pr}_{1}^{*}\left(\left[x_{0}\right] \cdot\left[x_{0}\right]\right)=p^{*} p_{*}\left(\left[x_{0}\right] \cdot\left[x_{0}\right]\right)=p^{*} p_{*} x_{0, *} x_{0}^{*}\left(\left[x_{0}\right]\right)=p^{*} x_{0}^{*}\left(\left[x_{0}\right]\right)=-\psi .
$$

Summarizing, we find $\iota^{*}\left(\mathscr{F}\left([C]_{(0)}\right)\right)=-K / 2-\left[x_{0}\right]-\psi / 2$.

Proof of Theorem 4.5. Suppose that we have proven statement (ii) and that $\psi \in \mathscr{T}(J)$. Consider the $\mathbb{Q}$-subalgebra of $(\mathrm{CH}(J), \cdot)$ generated by the classes $\left\{p_{i, j}\right\}$ and $\psi$. We denote it by $\mathscr{T}^{\prime}(J)$ and we have $\mathscr{T}^{\prime}(J) \subset \mathscr{T}(J)$. By definition $\mathscr{T}^{\prime}(J)$ is stable under the action of $e \in \mathfrak{s l}_{2}$. Now, statement (ii) shows that $\mathscr{T}^{\prime}(J)$ is also stable under the action of $f \in \mathfrak{s l}_{2}$. It then follows from Remark 3.7 that $\mathscr{T}^{\prime}(J)$ is stable under $\mathscr{F}$. In particular, the classes $\left\{[C]_{(j)}\right\}$ are in $\mathscr{T}^{\prime}(J)$. Since $\mathscr{T}(J)$ is the smallest $\mathbb{Q}$-subalgebra containing $[C]$ and stable under the $\mathfrak{s l}_{2}$-action, there is necessarily an equality $\mathscr{T}^{\prime}(J)=\mathscr{T}(J)$, which proves statement (i).

Statement (ii) follows essentially from [Pol07a, formula (2.9)]. We just need to translate the notation carefully. Following Polishchuk, we write $\eta:=K / 2+\left[x_{0}\right]+\psi / 2$, which by (4.2) is equal to $\iota^{*}(\theta)$. We also have $f=-\tilde{X}_{2,0}(C) / 2$ in his notation. Define operators $\tilde{p}_{i, j}$ on $\mathrm{CH}(J)$ by $\tilde{p}_{i, j}(\alpha):=p_{i, j} \cdot \alpha$. Then the fact that

$$
p_{i, j}=\mathscr{F}\left(\theta^{(j-i+2) / 2} \cdot[C]_{(j)}\right)=\mathscr{F}\left(\iota_{*}\left(\eta^{(j-i+2) / 2}\right)_{(j)}\right)
$$


is translated into

$$
\tilde{p}_{i, j}=\frac{1}{i !} \tilde{X}_{0, i}\left(\eta^{(j-i+2) / 2}\right) .
$$

We apply formula (2.9) in loc. cit. and find

$$
\begin{aligned}
{\left[f, \tilde{p}_{i, j}\right] } & =-\frac{1}{2 \cdot i !}\left[\tilde{X}_{2,0}(C), \tilde{X}_{0, i}\left(\eta^{(j-i+2) / 2}\right)\right] \\
& =\frac{1}{(i-1) !} \tilde{X}_{1, i-1}\left(\eta^{(j-i+2) / 2}\right)-\frac{1}{(i-2) !} \tilde{X}_{0, i-2}\left(\eta^{(j-i+4) / 2}\right) .
\end{aligned}
$$

Note that the second equality above also involves the fact that $\tilde{X}_{i, 0}(C)=0$ for $i \leqslant 1$ (see [Pol07a, Lemma 2.8]), and that $x_{0}^{*}(\eta)=x_{0}^{*} \iota^{*}(\theta)=o^{*}(\theta)=0$. We continue to calculate

$$
\begin{aligned}
{\left[\left[f, \tilde{p}_{i, j}\right], \tilde{p}_{k, l}\right]=} & \frac{1}{(i-1) ! k !}\left[\tilde{X}_{1, i-1}\left(\eta^{(j-i+2) / 2}\right), \tilde{X}_{0, k}\left(\eta^{(l-k+2) / 2}\right)\right] \\
& -\frac{1}{(i-2) ! k !}\left[\tilde{X}_{0, i-2}\left(\eta^{(j-i+4) / 2}\right), \tilde{X}_{0, k}\left(\eta^{(l-k+2) / 2}\right)\right] .
\end{aligned}
$$

Applying the same formula, we have $\left[\tilde{X}_{0, i-2}\left(\eta^{(j-i+4) / 2}\right), \tilde{X}_{0, k}\left(\eta^{(l-k+2) / 2}\right)\right]=0$ and

$$
\begin{aligned}
{\left[\tilde{X}_{1, i-1}\left(\eta^{(j-i+2) / 2}\right), \tilde{X}_{0, k}\left(\eta^{(l-k+2) / 2}\right)\right]=} & k \psi \tilde{X}_{0, k-1}\left(\eta^{(l-k+2) / 2}\right) \tilde{X}_{0, i-1}\left(\eta^{(j-i+2) / 2}\right) \\
& -k \tilde{X}_{0, i+k-2}\left(\eta^{(j-i+l-k+4) / 2}\right) .
\end{aligned}
$$

We thus obtain

$$
\begin{aligned}
{\left[\left[f, \tilde{p}_{i, j}\right], \tilde{p}_{k, l}\right]=} & \frac{1}{(i-1) !(k-1) !}\left(\psi \tilde{X}_{0, k-1}\left(\eta^{(l-k+2) / 2}\right) \tilde{X}_{0, i-1}\left(\eta^{(j-i+2) / 2}\right)\right. \\
& \left.-\tilde{X}_{0, i+k-2}\left(\eta^{(j-i+l-k+4) / 2}\right)\right) \\
= & \psi \tilde{p}_{k-1, l-1} \tilde{p}_{i-1, j-1}-\left(\begin{array}{c}
i+k-2 \\
i-1
\end{array}\right) \tilde{p}_{i+k-2, j+l} \\
= & \psi \tilde{p}_{i-1, j-1} \tilde{p}_{k-1, l-1}-\left(\begin{array}{c}
i+k-2 \\
i-1
\end{array}\right) \tilde{p}_{i+k-2, j+l}
\end{aligned}
$$

On the other hand, since $f([J])=0$, we have

$$
\left[f, \tilde{p}_{i, j}\right]([J])=f\left(p_{i, j}\right)=p_{i-2, j} .
$$

The identities (4.5) and (4.6) imply that for any polynomial $P$ in $\left\{p_{i, j}\right\}$ and $\psi$, we have

$$
f\left(P\left(\left\{p_{i, j}\right\}, \psi\right)\right)=\mathscr{D}\left(P\left(\left\{p_{i, j}\right\}, \psi\right)\right),
$$

where $\mathscr{D}$ is the differential operator defined in (4.1) (see [Pol07b, Section 3]).

It remains to prove that $\psi \in \mathscr{T}(J)$. To see this, we apply $\mathscr{D}$ to the class $p_{1,1}^{2} \in \mathscr{T}(J)$, which gives

$$
\mathscr{D}\left(p_{1,1}^{2}\right)=\psi p_{0,0}^{2}-\left(\begin{array}{l}
0 \\
0
\end{array}\right) p_{0,2}=g^{2} \psi-p_{0,2} .
$$

Hence $\psi=\left(\mathscr{D}\left(p_{1,1}^{2}\right)+p_{0,2}\right) / g^{2} \in \mathscr{T}(J)$.

Corollary 4.7. For $i \geqslant 0$, we have the identity

$$
p_{0,2 i}=\pi^{*}\left(\frac{1}{2^{i+1}} \sum_{0 \leqslant j \leqslant i}\left(\begin{array}{c}
i+1 \\
j+1
\end{array}\right) \psi^{i-j} \kappa_{j}+\psi^{i}\right) .
$$




\section{A TALE OF TWO TAUTOLOGICAL RINGS}

Moreover, we have the following isomorphisms of $\mathbb{Q}$-algebras (similar to those in (3.8)):

$$
\left(\bigoplus_{i=0}^{d} \mathscr{T}_{(0,2 i)}(J), \cdot\right) \underset{\pi^{*}}{\sim} \underset{(\mathscr{R}(S), \cdot)}{\sim} \underset{\pi_{*}}{\sim}\left(\bigoplus_{i=0}^{d} \mathscr{T}_{(2 g, 2 i)}(J), *\right)
$$

In particular, the ring $\mathscr{R}(S)$ may be regarded as a $\mathbb{Q}$-subalgebra of $(\mathscr{T}(J), \cdot)$ via $\pi^{*}$.

Proof. By the isomorphisms (3.8) we have

$$
p_{0,2 i}=\mathscr{F}\left(\theta^{i+1} \cdot[C]_{(2 i)}\right)=\pi^{*} \pi_{*}\left(\theta^{i+1} \cdot[C]_{(2 i)}\right)=\pi^{*} \pi_{*}\left(\theta^{i+1} \cdot[C]\right) .
$$

Hence it suffices to calculate $\pi_{*}\left(\theta^{i+1} \cdot[C]\right)$. Applying (4.2) and the projection formula, we get

$$
\begin{aligned}
\pi_{*}\left(\theta^{i+1} \cdot[C]\right) & =p_{*}\left(\left(\frac{1}{2} K+\left[x_{0}\right]+\frac{1}{2} \psi\right)^{i+1}\right) \\
& =\sum_{\substack{j+k+l=i+1 \\
j, k, l \geqslant 0}} \frac{(i+1) !}{j ! k ! l !} \frac{1}{2^{j+l}} p_{*}\left(K^{j} \cdot\left[x_{0}\right]^{k} \cdot \psi^{l}\right) .
\end{aligned}
$$

Applying the projection formula again, to $p: C \rightarrow S$ and $x_{0}: S \rightarrow C$, we find

$$
p_{*}\left(K^{j} \cdot\left[x_{0}\right]^{k} \cdot \psi^{l}\right)=\psi^{l} \cdot p_{*}\left(K^{j} \cdot\left[x_{0}\right]^{k}\right)= \begin{cases}\psi^{l} \cdot \kappa_{j-1} & \text { if } k=0, \\ \psi^{l} \cdot x_{0}^{*}\left(K^{j} \cdot\left[x_{0}\right]^{k-1}\right)=(-1)^{k-1} \psi^{i} & \text { if } k \geqslant 1,\end{cases}
$$

with the convention $\kappa_{-1}=0$. It follows that

$$
\begin{aligned}
\pi_{*}\left(\theta^{i+1} \cdot[C]\right)= & \sum_{\substack{j+l=i+1 \\
j, l \geqslant 0}} \frac{(i+1) !}{j ! l !} \frac{1}{2^{i+1}} \psi^{l} \kappa_{j-1}+\sum_{\substack{j+k+l=i+1 \\
j, k, l \geqslant 0}} \frac{(i+1) !}{j ! k ! l !} \frac{1}{2^{j+l}}(-1)^{k-1} \psi^{i} \\
& -\sum_{\substack{j+l=i+1 \\
j, l \geqslant 0}} \frac{(i+1) !}{j ! l !} \frac{1}{2^{i+1}}(-1) \psi^{i} \\
= & \frac{1}{2^{i+1}} \sum_{0 \leqslant j \leqslant i}\left(\begin{array}{l}
i+1 \\
j+1
\end{array}\right) \psi^{i-j} \kappa_{j}+\left(\frac{1}{2}-1+\frac{1}{2}\right)^{i+1} \psi^{i}+\left(\frac{1}{2}+\frac{1}{2}\right)^{i+1} \psi^{i} \\
= & \frac{1}{2^{i+1}} \sum_{0 \leqslant j \leqslant i}\left(\begin{array}{c}
i+1 \\
j+1
\end{array}\right) \psi^{i-j} \kappa_{j}+\psi^{i},
\end{aligned}
$$

which proves the identity (4.7).

Now since $\oplus_{i=0}^{d} \mathscr{T}_{(0,2 i)}(J)$ is generated by the classes $\left\{p_{0,2 i}\right\}$ and $\psi$, we have one inclusion $\oplus_{i=0}^{d} \mathscr{T}_{(0,2 i)}(J) \subset \pi^{*}(\mathscr{R}(S))$. For the other inclusion, it follows from (4.7) and induction on $j$ that one can also express $\pi^{*}\left(\kappa_{j}\right)$ as linear combinations of $\left\{p_{0,2 i}\right\}$ and $\psi$. So we have $\oplus_{i=0}^{d} \mathscr{T}_{(0,2 i)}(J)=$ $\pi^{*}(\mathscr{R}(S))$, and the rest follows from (3.8).

\section{The Chow rings of the relative infinite symmetric power}

A nice way to treat the Chow rings of all $C^{[n]}$ simultaneously is to consider the relative infinite symmetric power $C^{[\infty]}$. It allows us to lift structures from the Chow ring of $J$. We recall the definition of $C^{[\infty]}$ and its Chow theories. Our reference is the paper of Moonen and Polishchuk [MP10], which generalizes the work of Kimura and Vistoli [KV96] to the relative setting. 
5.1. We retain the notation of Sections 2 and 3. For $n \geqslant 1$, define maps $\varphi_{n}: C^{[n]} \rightarrow J$ and $\phi_{n}:=\varphi_{n} \circ \sigma_{n}: C^{n} \rightarrow J$, which send (locally) $n$ sections $x_{1}, \ldots, x_{n}$ of $C / S$ to the class $\mathscr{O}_{C}\left(x_{1}+\right.$ $\left.\cdots+x_{n}-n x_{0}\right)$. Note that $\varphi_{1}=\phi_{1}=\iota: C \hookrightarrow J$. For convenience we set $\varphi_{0}=\phi_{0}:=o: S \rightarrow J$. We have the following diagram in addition to (3.1):

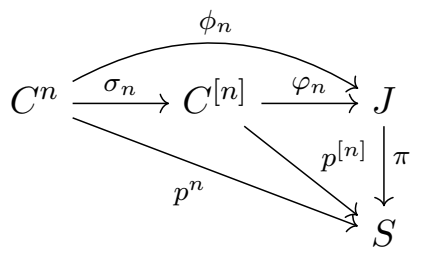

5.2. We introduce the relative infinite symmetric power of $C$, which is defined to be the indscheme

$$
C^{[\infty]}:=\underline{\lim }\left(S=C^{[0]} \hookrightarrow C \hookrightarrow C^{[2]} \hookrightarrow C^{[3]} \hookrightarrow \cdots\right) .
$$

Here the transition maps $\varepsilon_{n}: C^{[n-1]} \hookrightarrow C^{[n]}$ are given by adding a copy of $x_{0}$ (in particular, $\left.\varepsilon_{1}=x_{0}: S \rightarrow C\right)$. We write $p^{[\infty]}: C^{[\infty]} \rightarrow S$ for the limit of $p^{[n]}: C^{[n]} \rightarrow S$.

The collection of $\varphi_{n}: C^{[n]} \rightarrow J$ induces $\varphi: C^{[\infty]} \rightarrow J$. Moreover, the monoidal structure on $J$ can be lifted to $C^{[\infty]}$ : the addition maps $\mu_{n, m}: C^{[n]} \times{ }_{S} C^{[m]} \rightarrow C^{[n+m]}$ give rise to

$$
\mu: C^{[\infty]} \times{ }_{S} C^{[\infty]} \rightarrow C^{[\infty]}
$$

while the diagonal maps $\Delta_{N}: C^{[n]} \rightarrow C^{[N n]}$ give rise to

$$
[N]: C^{[\infty]} \rightarrow C^{[\infty]} \text { for } N \geqslant 0 .
$$

Unlike the case of $J$, there are (at least) two different notions of Chow ring for $C^{[\infty]}$. One is graded by codimension and the other by relative dimension. For this reason, we also distinguish the two gradings on $\mathrm{CH}(J)$ : we write $\mathrm{CH}^{\bullet}(J):=\left(\oplus_{i} \mathrm{CH}^{i}(J), \cdot\right)$ and $\mathrm{CH}_{\bullet}(J):=\left(\oplus_{i} \mathrm{CH}_{i}(J), *\right)$.

Definition 5.3. (i) The Chow cohomology of $C^{[\infty]}$ of codimension $i$ is the inverse limit

$$
\mathrm{CH}^{i}\left(C^{[\infty]}\right):=\lim \left(\mathrm{CH}^{i}(S) \leftarrow \mathrm{CH}^{i}(C) \leftarrow \mathrm{CH}^{i}\left(C^{[2]}\right) \leftarrow \mathrm{CH}^{i}\left(C^{[3]}\right) \leftarrow \cdots\right),
$$

where the transition maps are $\varepsilon_{n}^{*}: \mathrm{CH}^{i}\left(C^{[n]}\right) \rightarrow \mathrm{CH}^{i}\left(C^{[n-1]}\right)$.

(ii) The Chow homology of $C^{[\infty]}$ of relative dimension $i$ is the direct limit

$$
\mathrm{CH}_{i}\left(C^{[\infty]}\right):=\lim _{\longrightarrow}\left(\mathrm{CH}_{i}(S) \rightarrow \mathrm{CH}_{i}(C) \rightarrow \mathrm{CH}_{i}\left(C^{[2]}\right) \rightarrow \mathrm{CH}_{i}\left(C^{[3]}\right) \rightarrow \cdots\right),
$$

where the transition maps are $\varepsilon_{n, *}: \mathrm{CH}_{i}\left(C^{[n-1]}\right) \rightarrow \mathrm{CH}_{i}\left(C^{[n]}\right)$.

By definition, an element in $\mathrm{CH}^{i}\left(C^{[\infty]}\right)$ is a sequence $\alpha=\left(\alpha_{n}\right)_{n \geqslant 0}$ with $\alpha_{n} \in \mathrm{CH}^{i}\left(C^{[n]}\right)$ such that $\varepsilon_{n}^{*}\left(\alpha_{n}\right)=\alpha_{n-1}$. An element in $\mathrm{CH}_{i}\left(C^{[\infty]}\right)$ is represented by $\alpha \in \mathrm{CH}_{i}\left(C^{[n]}\right)$ for some $n$. We write $\mathrm{CH}^{\bullet}\left(C^{[\infty]}\right):=\oplus_{i} \mathrm{CH}^{i}\left(C^{[\infty]}\right)$ and $\mathrm{CH}_{\bullet}\left(C^{[\infty]}\right):=\oplus_{i} \mathrm{CH}_{i}\left(C^{[\infty]}\right)$. The Chow cohomology and homology of $C^{[\infty]} \times{ }_{S} C^{[\infty]}$ are defined similarly.

Remark 5.4. For $n \geqslant 1$, the transition map $\varepsilon_{n}^{*}$ (respectively, $\varepsilon_{n, *}$ ) is surjective (respectively, injective). To see this, one constructs a correspondence $\delta_{n}: C^{[n]} \vdash C^{[n-1]}$ satisfying $\delta_{n} \circ \varepsilon_{n}=\mathrm{id}$, so that $\varepsilon_{n}^{*} \circ \delta_{n}^{*}=$ id and $\delta_{n, *} \circ \varepsilon_{n, *}=$ id. Let $\operatorname{pr}_{i}: C^{n} \rightarrow C$ be the $i$ th projection. Consider the correspondence (see [KV96, Definition 1.8])

$$
\sum_{j=0}^{n-1} \sum_{1 \leqslant i_{1}<\cdots<i_{j} \leqslant n}(-1)^{n-1-j}\left(\operatorname{pr}_{i_{1}}, \ldots, \operatorname{pr}_{i_{j}}, x_{0}, \ldots, x_{0}\right): C^{n} \vdash C^{n-1},
$$




\section{A tale of TWO TAUTOLOGICAL RINGS}

which is $\mathfrak{S}_{n}$-invariant and descends to $\delta_{n}: C^{[n]} \vdash C^{[n-1]}$. One verifies that $\delta_{n}$ has the desired property. In particular, the map $\mathrm{CH}^{\bullet}\left(C^{[\infty]}\right) \rightarrow \mathrm{CH}\left(C^{[n]}\right)\left(\operatorname{respectively,~} \mathrm{CH}\left(C^{[n]}\right) \rightarrow \mathrm{CH}_{\bullet}\left(C^{[\infty]}\right)\right.$ ) is surjective (respectively, injective).

5.5. Both $\mathrm{CH}^{\bullet}\left(C^{[\infty]}\right)$ and $\mathrm{CH}_{\bullet}\left(C^{[\infty]}\right)$ are equipped with a ring structure: there is the intersection product $\cdot$ on $\mathrm{CH}^{\bullet}\left(C^{[\infty]}\right)$ and the Pontryagin product $*$ on $\mathrm{CH}_{\bullet}\left(C^{[\infty]}\right)$, the latter defined in the same way as (3.2).

Note that unlike the case of $\mathrm{CH}(J)$, one cannot define both ring structures on the same object $\mathrm{CH}^{\bullet}\left(C^{[\infty]}\right)$ or $\mathrm{CH}_{\bullet}\left(C^{[\infty]}\right)$. We do, however, have a cap product

$$
\mathrm{CH}^{\bullet}\left(C^{[\infty]}\right) \times \mathrm{CH}_{\bullet}\left(C^{[\infty]}\right) \stackrel{\cap}{\rightarrow} \mathrm{CH}_{\bullet}\left(C^{[\infty]}\right),
$$

which sends $\alpha=\left(\alpha_{n}\right)_{n \geqslant 0} \in \mathrm{CH}^{i}\left(C^{[\infty]}\right)$ and $\beta \in \mathrm{CH}_{j}\left(C^{[m]}\right) \subset \mathrm{CH}_{j}\left(C^{[\infty]}\right)$ to $\alpha \cap \beta:=\alpha_{m} \cdot \beta \in$ $\mathrm{CH}_{j-i}\left(C^{[m]}\right) \subset \mathrm{CH}_{j-i}\left(C^{[\infty]}\right)$. One verifies that $\cap$ is well defined.

For $N \geqslant 0$, the multiplication $[N]: C^{[\infty]} \rightarrow C^{[\infty]}$ induces

$$
[N]^{*}: \mathrm{CH}^{\bullet}\left(C^{[\infty]}\right) \rightarrow \mathrm{CH}^{\bullet}\left(C^{[\infty]}\right) \text { and }[N]_{*}: \mathrm{CH}_{\bullet}\left(C^{[\infty]}\right) \rightarrow \mathrm{CH}_{\bullet}\left(C^{[\infty]}\right) .
$$

Further, the map $\varphi: C^{[\infty]} \rightarrow J$ induces morphisms of $\mathbb{Q}$-algebras

$$
\varphi^{*}: \mathrm{CH}^{\bullet}(J) \rightarrow \mathrm{CH}^{\bullet}\left(C^{[\infty]}\right) \text { and } \varphi_{*}: \mathrm{CH}_{\bullet}\left(C^{[\infty]}\right) \rightarrow \mathrm{CH}_{\bullet}(J),
$$

which allow us to relate the Chow theories of $C^{[\infty]}$ and $J$. We begin with $\mathrm{CH}^{\bullet}\left(C^{[\infty]}\right)$.

5.6. Chow cohomology. Recall the class $\psi \in \mathrm{CH}^{1}(S)$. Again we keep the same notation $\psi$ for its pull-backs to schemes over $S$. For $n \geqslant 1$, define

$$
\mathscr{O}_{C^{[n]}}(1):=\mathscr{O}_{C}^{[n]}\left(\varepsilon_{n}\left(C^{[n-1]}\right)+n \psi\right),
$$

and denote by $\xi_{n} \in \mathrm{CH}^{1}\left(C^{[n]}\right)$ the first Chern class of $\mathscr{O}_{C}^{[n]}(1)$. We set $\xi_{0}=0$. Then we have $\varepsilon_{n}^{*}\left(\xi_{n}\right)=\xi_{n-1}$, which yields a class

$$
\xi:=\left(\xi_{n}\right)_{n \geqslant 0} \in \mathrm{CH}^{1}\left(C^{[\infty]}\right) .
$$

There is an alternative description of $\mathscr{O}_{C[n]}(1)$ (essentially due to Schwarzenberger [Sch63]). Let $\mathscr{L}$ be the pull-back of the Poincaré line bundle $\mathscr{P}$ via $\iota \times_{S} \operatorname{id}{ }_{J}: C \times_{S} J \rightarrow J \times{ }_{S} J$. For $n \geqslant 0$, define the sheaf

$$
E_{n}:=\operatorname{pr}_{2, *}\left(\operatorname{pr}_{1}^{*}\left(\mathscr{O}_{C}\left(n x_{0}\right)\right) \otimes \mathscr{L}\right),
$$

where $\operatorname{pr}_{1}: C \times_{S} J \rightarrow C$ and $\mathrm{pr}_{2}: C \times_{S} J \rightarrow J$ are the two projections. There is a canonical isomorphism $C^{[n]} \simeq \mathbb{P}\left(E_{n}\right)$, under which $\mathscr{O}_{C}^{[n]}(1)$ corresponds to the line bundle $\mathscr{O}_{\mathbb{P}\left(E_{n}\right)}(1)$.

When $n \geqslant 2 g-1$, the sheaf $E_{n}$ is locally free over $J$ (that is, a vector bundle). Then we have isomorphisms of $\mathbb{Q}$-algebras

$$
\mathrm{CH}\left(C^{[n]}\right) \simeq \mathrm{CH}\left(\mathbb{P}\left(E_{n}\right)\right) \simeq \mathrm{CH}(J)\left[\xi_{n}\right] /\left\langle P\left(\xi_{n}\right)\right\rangle,
$$

where $P\left(\xi_{n}\right):=\sum_{i=0}^{n-g+1} c_{n-g+1-i}\left(E_{n}\right) \xi_{n}^{i}$ is a polynomial in $\xi_{n}$ of degree $n-g+1$ with coefficients in $\mathrm{CH}(J)$.

5.7. The following results are merely reinterpretations of (5.4) [MP10, Theorem 1.4]:

(i) When $n \geqslant \max \{2 g, i+g+1\}$, the transition map $\varepsilon_{n}^{*}: \mathrm{CH}^{i}\left(C^{[n]}\right) \rightarrow \mathrm{CH}^{i}\left(C^{[n-1]}\right)$ becomes an isomorphism. 
Q. Y IN

(ii) The map $\varphi^{*}: \mathrm{CH}^{\bullet}(J) \rightarrow \mathrm{CH}^{\bullet}\left(C^{[\infty]}\right)$ induces an isomorphism of $\mathbb{Q}$-algebras

$$
\Phi: \mathrm{CH}^{\bullet}(J)[t] \stackrel{\sim}{\rightarrow} \mathrm{CH}^{\bullet}\left(C^{[\infty]}\right),
$$

which sends $\alpha \in \mathrm{CH}^{\bullet}(J)$ to $\varphi^{*}(\alpha)$ and $t$ to the class $\xi$.

5.8. Chow homology. We switch to $\mathrm{CH}_{\bullet}\left(C^{[\infty]}\right)$. First, we choose an integer $n \geqslant 2 g+d$ (recall that $d=\operatorname{dim}(S))$, and we identify $C^{[n]}$ with $\mathbb{P}\left(E_{n}\right)$. Consider the short exact sequence

$$
0 \rightarrow \mathscr{O}_{C[n]}(-1) \rightarrow \varphi_{n}^{*}\left(E_{n}\right) \rightarrow Q \rightarrow 0,
$$

where $Q$ is the universal quotient bundle of $\varphi_{n}^{*}\left(E_{n}\right)$ and is of rank $n-g$. Define

$$
\Gamma:=c_{n-g}(Q) \in \mathrm{CH}_{g}\left(C^{[n]}\right) \subset \mathrm{CH}_{g}\left(C^{[\infty]}\right) .
$$

Next, choose $n \geqslant 2 g+d+1$. Define

$$
L:=\varphi_{n}^{*}([o]) \cdot c_{n-g-1}(Q) \in \mathrm{CH}_{1}\left(C^{[n]}\right) \subset \mathrm{CH}_{1}\left(C^{[\infty]}\right),
$$

where $[o]:=[o(S)] \in \mathrm{CH}_{0}(J)$ is the class of the zero section. One can show that both classes $\Gamma \in \mathrm{CH}_{g}\left(C^{[\infty]}\right)$ and $L \in \mathrm{CH}_{1}\left(C^{[\infty]}\right)$ are independent of $n$.

5.9. Here are the corresponding results for $\mathrm{CH}_{\bullet}\left(C^{[\infty]}\right)[\mathrm{MP} 10$, Theorem 1.11]:

(i) The map $s: \mathrm{CH}_{\bullet}(J) \rightarrow \mathrm{CH}_{\bullet}\left(C^{[\infty]}\right)$ given by

$$
s(\alpha):=\varphi^{*}(\alpha) \cap \Gamma
$$

is a section of $\varphi_{*}: \mathrm{CH}_{\bullet}\left(C^{[\infty]}\right) \rightarrow \mathrm{CH}_{\bullet}(J)$. It respects the Pontryagin products on both sides.

(ii) The section $s$ induces an isomorphism of $\mathbb{Q}$-algebras

$$
\Psi: \mathrm{CH}_{\bullet}(J)[t] \stackrel{\sim}{\rightarrow} \mathrm{CH}_{\bullet}\left(C^{[\infty]}\right),
$$

which sends $\alpha \in \mathrm{CH}_{\bullet}(J)$ to $s(\alpha)$ and $t$ to the class $L$.

(iii) Under the isomorphism $\Psi$, the push-forward $\varphi_{*}$ is the evaluation at zero and the action of $\xi \cap$ is the derivation $\mathrm{d} / \mathrm{d} t$.

Remark 5.10. There are explicit expressions for $\Gamma$ and $L$ (see [MP10, Corollary 1.13]):

$$
\Gamma=\frac{1}{g !(N-1)^{g}}\left(\frac{\log \left(1+\psi \cdot[N]_{*}([C])\right)-N \log (1+\psi \cdot[C])}{N \psi}\right)^{g},
$$

with $N \geqslant 2$, and

$$
L=\frac{\log (1+\psi \cdot[C])-\log (1+\psi \cdot s([\iota(C)]))}{\psi} .
$$

Here we distinguish $[C] \in \mathrm{CH}_{1}(C) \subset \mathrm{CH}_{1}\left(C^{[\infty]}\right)$ from $[\iota(C)] \in \mathrm{CH}_{1}(J)$.

5.11. Fourier transform. By [KV96, Theorems 3.13 and 3.18], the Fourier transform on $J$ can be lifted to $C^{[\infty]}$. Recall the class $\ell \in \mathrm{CH}^{1}\left(J \times_{S} J\right)$. Define

$$
\begin{aligned}
\ell_{\infty, \infty} & :=\left(\varphi \times \times_{S} \varphi\right)^{*}(\ell) \in \mathrm{CH}^{1}\left(C^{[\infty]} \times_{S} C^{[\infty]}\right), \\
\xi \times{ }_{S} \xi & :=\operatorname{pr}_{1}^{*}(\xi) \cdot \operatorname{pr}_{2}^{*}(\xi) \in \mathrm{CH}^{2}\left(C^{[\infty]} \times{ }_{S} C^{[\infty]}\right),
\end{aligned}
$$

where $\operatorname{pr}_{1}, \operatorname{pr}_{2}: C^{[\infty]} \times{ }_{S} C^{[\infty]} \rightarrow C^{[\infty]}$ are the two projections. Then the expression

$$
\exp \left(\ell_{\infty, \infty}+\xi \times_{S} \xi\right)
$$




\section{A TALE OF TWO TAUTOLOGiCAL RiNGS}

is an upper correspondence in the sense of [KV96, Definition 3.2]. It induces an isomorphism of $\mathbb{Q}$-algebras

$$
\mathscr{F}: \mathrm{CH}_{\bullet}\left(C^{[\infty]}\right) \stackrel{\sim}{\rightarrow} \mathrm{CH}^{\bullet}\left(C^{[\infty]}\right) .
$$

We have $\mathscr{F}(L)=\xi$, and the following commutative diagram:

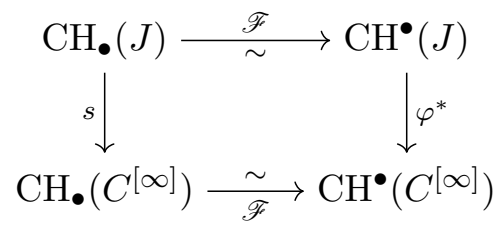

Further, the inverse $\mathscr{F}^{-1}: \mathrm{CH}^{\bullet}\left(C^{[\infty]}\right) \stackrel{\sim}{\rightarrow} \mathrm{CH}_{\bullet}\left(C^{[\infty]}\right)$ is given by the lower correspondence in the sense of [KV96, Definition 3.17]:

$$
(-1)^{g} \exp \left(-\ell_{\infty, \infty}\right) \cap\left(\exp ^{*}\left(\left(L \times_{S} L\right) *\left(\Gamma \times_{S} \Gamma\right)\right)\right),
$$

where exp* means the exponential power series with respect to $*$.

Remark 5.12. The class $\ell_{\infty, \infty}$ can be described somewhat explicitly. For $n, m \geqslant 0$, define

$$
\ell_{n, m}:=\left(\varphi_{n} \times{ }_{S} \varphi_{m}\right)^{*}(\ell) \in \mathrm{CH}^{1}\left(C^{[n]} \times_{S} C^{[m]}\right) .
$$

We have $\ell_{n, 0}=\ell_{0, m}=0$, and we have seen in (4.3) that

$$
\ell_{1,1}=[\Delta]-\operatorname{pr}_{1}^{*}\left(\left[x_{0}\right]\right)-\operatorname{pr}_{2}^{*}\left(\left[x_{0}\right]\right)-\psi \quad \text { in } \mathrm{CH}^{1}\left(C \times_{S} C\right) .
$$

For $1 \leqslant i \leqslant n$, let $\operatorname{pr}_{i}: C^{n} \rightarrow C$ be the $i$ th projection. Then for $n, m \geqslant 1$, there is an identity (see [KV96, Proposition-Definition 3.10])

$$
\left(\phi_{n} \times_{S} \phi_{m}\right)^{*}(\ell)=\left(\sigma_{n} \times_{S} \sigma_{m}\right)^{*}\left(\ell_{n, m}\right)=\sum_{i=1}^{n} \sum_{j=1}^{m}\left(\operatorname{pr}_{i} \times_{S} \operatorname{pr}_{j}\right)^{*}\left(\ell_{1,1}\right),
$$

which holds in $\mathrm{CH}^{1}\left(C^{n} \times{ }_{S} C^{m}\right)^{\mathfrak{S}_{n} \times \mathfrak{S}_{m}}$.

\section{The tautological rings of the relative infinite symmetric power}

We define the tautological cohomology $\mathscr{R}^{\bullet}\left(C^{[\infty]}\right)$ and homology $\mathscr{R}_{\bullet}\left(C^{[\infty]}\right)$ of the relative infinite symmetric power. We prove a tautological analog of the isomorphisms $\Phi$ and $\Psi$ in the previous section, that both $\mathscr{R}^{\bullet}\left(C^{[\infty]}\right)$ and $\mathscr{R} \bullet\left(C^{[\infty]}\right)$ are polynomial algebras over $\mathscr{T}(J)$. The connections between the two notions of tautological ring are thus established. Throughout this section we work in the setting of (3.1) and (5.1).

6.1. First, observe that the rings $\mathscr{R}\left(C^{[n]}\right)$ are stable under pull-backs and push-forwards via the maps $\varepsilon_{n}: C^{[n-1]} \hookrightarrow C^{[n]}$. In fact, the maps $\varepsilon_{n}$ can be lifted to $\operatorname{id}_{C^{n-1}} \times_{S} x_{0}: C^{n-1} \rightarrow C^{n}$. Then for $\alpha \in \mathscr{R}\left(C^{n}\right)$ and $\beta \in \mathscr{R}\left(C^{n-1}\right)$, we have

$$
\begin{aligned}
& \left(\operatorname{id}_{C^{n-1}} \times_{S} x_{0}\right)^{*}(\alpha)=\operatorname{pr}_{1, \ldots, n-1, *}\left(\alpha \cdot \operatorname{pr}_{n}^{*}\left(\left[x_{0}\right]\right)\right) \in \mathscr{R}\left(C^{n-1}\right), \\
& \left(\operatorname{id}_{C^{n-1}} \times_{S} x_{0}\right)_{*}(\beta)=\operatorname{pr}_{1, \ldots, n-1}^{*}(\beta) \cdot \operatorname{pr}_{n}^{*}\left(\left[x_{0}\right]\right) \in \mathscr{R}\left(C^{n}\right) .
\end{aligned}
$$

Here $\operatorname{pr}_{1, \ldots, n-1}\left(\right.$ respectively, $\mathrm{pr}_{n}$ ) is the projection of $C^{n}$ to the first $n-1$ factors (respectively, $n$th factor), and is tautological in the sense of Remark 2.4.

The stability of $\mathscr{R}\left(C^{[n]}\right)$ under $\varepsilon_{n}^{*}$ and $\varepsilon_{n, *}$ allows us to pass to $C^{[\infty]}$. 
Definition 6.2. (i) The tautological cohomology of $C^{[\infty]}$ is the inverse limit

$$
\mathscr{R} \bullet\left(C^{[\infty]}\right):=\lim _{\longleftarrow}\left(\mathscr{R}(S) \leftarrow \mathscr{R}(C) \leftarrow \mathscr{R}\left(C^{[2]}\right) \leftarrow \mathscr{R}\left(C^{[3]}\right) \leftarrow \cdots\right),
$$

where the transition maps are $\varepsilon_{n}^{*}: \mathscr{R}\left(C^{[n]}\right) \rightarrow \mathscr{R}\left(C^{[n-1]}\right)$.

(ii) The tautological homology of $C^{[\infty]}$ is the direct limit

$$
\mathscr{R} \bullet\left(C^{[\infty]}\right):=\underline{\lim }\left(\mathscr{R}(S) \rightarrow \mathscr{R}(C) \rightarrow \mathscr{R}\left(C^{[2]}\right) \rightarrow \mathscr{R}\left(C^{[3]}\right) \rightarrow \cdots\right),
$$

where the transition maps are $\varepsilon_{n, *}: \mathscr{R}\left(C^{[n-1]}\right) \rightarrow \mathscr{R}\left(C^{[n]}\right)$.

Note that $\mathscr{R}^{\bullet}\left(C^{[\infty]}\right)$ (respectively, $\left.\mathscr{R} \bullet\left(C^{[\infty]}\right)\right)$ inherits a grading from $\mathrm{CH}^{\bullet}\left(C^{[\infty]}\right)$ (respectively, $\left.\mathrm{CH}_{\bullet}\left(C^{[\infty]}\right)\right)$. It is immediate that $\mathscr{R}^{\bullet}\left(C^{[\infty]}\right)$ is stable under the intersection product $\cdot$. Since the addition map $\mu_{n, m}: C^{[n]} \times_{S} C^{[m]} \rightarrow C^{[n+m]}$ lifts to the identity (tautological) map $C^{n} \times{ }_{S}$ $C^{m} \rightarrow C^{n+m}$, we also know that $\mathscr{R}_{\bullet}\left(C^{[\infty]}\right)$ is stable under the Pontryagin product $*$. It follows that $\mathscr{R}^{\bullet}\left(C^{[\infty]}\right)$ (respectively, $\mathscr{R} \bullet\left(C^{[\infty]}\right)$ ) is a graded $\mathbb{Q}$-subalgebra of $\mathrm{CH}^{\bullet}\left(C^{[\infty]}\right)$ (respectively, $\left.\mathrm{CH}_{\bullet}\left(C^{[\infty]}\right)\right)$.

We list a few properties of $\mathscr{R}^{\bullet}\left(C^{[\infty]}\right)$ and $\mathscr{R} \bullet\left(C^{[\infty]}\right)$, which partly reveal their links with the $\operatorname{ring} \mathscr{T}(J)$.

Proposition 6.3. (i) We have $\xi \in \mathscr{R}^{1}\left(C^{[\infty]}\right), \Gamma \in \mathscr{R}_{g}\left(C^{[\infty]}\right)$ and $L \in \mathscr{R}_{1}\left(C^{[\infty]}\right)$.

(ii) The ring $\mathscr{R}^{\bullet}\left(C^{[\infty]}\right)$ (respectively, $\mathscr{R} \bullet\left(C^{[\infty]}\right)$ ) is stable under $[N]^{*}$ (respectively, $\left.[N]_{*}\right)$ for all $N \geqslant 0$.

(iii) The Fourier transform $\mathscr{F}$ induces an isomorphism

$$
\mathscr{F}: \mathscr{R} \bullet\left(C^{[\infty]}\right) \stackrel{\sim}{\rightarrow} \mathscr{R} \bullet\left(C^{[\infty]}\right) .
$$

(iv) The cap product restricts to a map

$$
\mathscr{R} \bullet\left(C^{[\infty]}\right) \times \mathscr{R} \bullet\left(C^{[\infty]}\right) \stackrel{\cap}{\rightarrow} \mathscr{R} \bullet\left(C^{[\infty]}\right) .
$$

Proof. Statement (ii) follows from the fact that the diagonal map $\Delta_{N}: C^{[n]} \rightarrow C^{[N n]}$ lifts to $C^{n} \rightarrow C^{N n}$, which is tautological. Statement (iv) is straightforward.

For statement (i), by (5.3) and (5.7) we have $\xi \in \mathscr{R}^{1}\left(C^{[\infty]}\right)$ and $\Gamma \in \mathscr{R}_{g}\left(C^{[\infty]}\right)$. Moreover, by (5.8), to prove that $L \in \mathscr{R}_{1}\left(C^{[\infty]}\right)$ it suffices to show that $s([\iota(C)]) \in \mathscr{R}_{1}\left(C^{[\infty]}\right)$. This is further reduced to proving that $\varphi^{*}([\iota(C)]) \in \mathscr{R}^{g-1}\left(C^{[\infty]}\right)$ by the definition of the section $s$.

In fact, we can prove for any $\alpha \in \mathscr{T}(J)$ that $\varphi^{*}(\alpha) \in \mathscr{R}^{\bullet}\left(C^{[\infty]}\right)$. First, by Theorem 4.5, we know that $(\mathscr{T}(J), \cdot)$ is generated by the classes $\left\{p_{i, j}\right\}$ and $\psi$. Since $\varphi^{*}(\psi) \in \mathscr{R}^{1}\left(C^{[\infty]}\right)$, it remains to prove that $\varphi^{*}\left(p_{i, j}\right) \in \mathscr{R}^{\bullet}\left(C^{[\infty]}\right)$ for all possible $i$ and $j$. Here we can actually calculate the pull-back of $p_{i, j}$ via $\phi_{n}=\varphi_{n} \circ \sigma_{n}: C^{n} \rightarrow J$, for all $n \geqslant 0$. The procedure is similar to that of Lemma 4.6: we chase through the following cartesian squares:

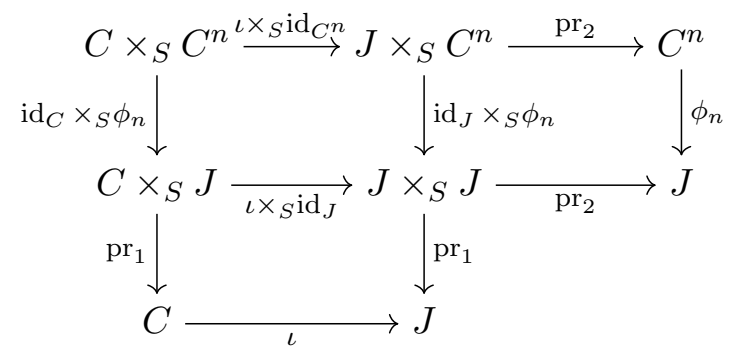




\section{A tAlE OF TWO TAUTOLOGICAL RINGS}

Then we find

$$
\phi_{n}^{*}\left(\mathscr{F}\left(\theta^{(j-i+2) / 2} \cdot[\iota(C)]\right)\right)=\operatorname{pr}_{2, *}\left(\operatorname{pr}_{1}^{*}\left(\iota^{*}(\theta)^{(j-i+2) / 2}\right) \cdot \exp \left(\left(\iota \times_{S} \phi_{n}\right)^{*}(\ell)\right)\right),
$$

where $\mathrm{pr}_{1}: C \times{ }_{S} C^{n} \rightarrow C$ and $\mathrm{pr}_{2}: C \times{ }_{S} C^{n} \rightarrow C^{n}$ are the two projections. By definition $\phi_{n}^{*}\left(p_{i, j}\right)$ is just the codimension $(i+j) / 2$ component of the right-hand side of (6.1). Further, by (4.2) and (5.11), we have explicit expressions for $\iota^{*}(\theta)$ and $\left(\iota \times_{S} \phi_{n}\right)^{*}(\ell)$ in terms of tautological classes. It follows that $\phi_{n}^{*}\left(p_{i, j}\right) \in \mathscr{R}\left(C^{n}\right)$, and hence $\varphi^{*}\left(p_{i, j}\right) \in \mathscr{R} \bullet\left(C^{[\infty]}\right)$.

Finally, to prove statement (iii), we observe that the correspondences in (5.9) and (5.10) that define $\mathscr{F}$ and $\mathscr{F}^{-1}$ involve only tautological classes.

Now, we state and prove the main result of this section. To be coherent, we write $\mathscr{T}^{\bullet}(J):=$ $\left(\oplus_{i} \mathscr{T}^{i}(J), \cdot\right)$ and $\mathscr{T}_{\bullet}(J):=\left(\oplus_{i} \mathscr{T}_{i}(J), *\right)$.

TheOREM 6.4. The isomorphisms $\Phi$ and $\Psi$ in (5.5) and (5.6) restrict to isomorphisms of $\mathbb{Q}$ algebras

$$
\begin{gathered}
\Phi_{\mathscr{T} \bullet(J)[t]}: \mathscr{T} \bullet(J)[t] \stackrel{\sim}{\rightarrow} \mathscr{R} \bullet\left(C^{[\infty]}\right), \\
\left.\Psi\right|_{\mathscr{T}(J)[t]}: \mathscr{T}_{\bullet}(J)[t] \stackrel{\sim}{\rightarrow} \mathscr{R} \bullet\left(C^{[\infty]}\right) .
\end{gathered}
$$

The plan is to prove (6.3) first, and then deduce (6.2) by Fourier duality. We begin with an elementary lemma.

Lemma 6.5. Let $A$ be a commutative $\mathbb{Q}$-algebra, and let $B$ be a $\mathbb{Q}$-subalgebra of the polynomial algebra $A[t]$. Assume $t \in B$ and that $B$ is stable under the derivation $\mathrm{d} / \mathrm{d} t$. Then we have

$$
B=\operatorname{ev}(B)[t],
$$

where ev: $A[t] \rightarrow A$ is the evaluation at zero.

Proof. Take an element $P(t)=b_{0}+b_{1} t+\cdots+b_{n} t^{n}$ in $B$. Since $B$ is stable under derivation, we have $(\mathrm{d} / \mathrm{d} t)^{n}(P(t))=n ! b_{n} \in B$, so that $b_{n} \in B$. Then, since $t \in B$, we have $b_{n} t^{n} \in B$ and $P(t)-b_{n} t^{n} \in B$. By induction, we find that all coefficients $b_{i}$ are in $B$. It follows that $\mathrm{ev}(B)[t] \subset B$. On the other hand, we know that $b_{i}=\mathrm{ev}\left((\mathrm{d} / \mathrm{d} t)^{i}(P(t) / i !)\right)$ with $(\mathrm{d} / \mathrm{d} t)^{i}(P(t) / i !) \in B$. Hence $b_{i} \in \operatorname{ev}(B)$, which proves the other inclusion $B \subset \operatorname{ev}(B)[t]$.

Consider the push-forward map $\varphi_{*}: \mathrm{CH}_{\bullet}\left(C^{[\infty]}\right) \rightarrow \mathrm{CH}_{\bullet}(J)$ which, under $\Psi$, corresponds to the evaluation at zero. The proof of the following proposition is a bit involved and relies essentially on Theorem 4.5 and Corollary 4.7.

Proposition 6.6. We have $\varphi_{*}\left(\mathscr{R}_{\bullet}\left(C^{[\infty]}\right)\right)=\mathscr{T}_{\bullet}(J)$.

Proof. By Theorem 4.5, we know that $\mathscr{T}_{\bullet}(J)$ is generated by $\left\{\theta^{(j-i+2) / 2} \cdot[\iota(C)]_{(j)}\right\}$ and $o_{*}(\psi)$ (recall that $o=\varphi_{0}$ is the zero section). Consider the class $\eta=K / 2+\left[x_{0}\right]+\psi / 2 \in \mathscr{R}(C)$, which by (4.2) is equal to $\iota^{*}(\theta)$. We have

$$
\iota_{*}\left(\eta^{(j-i+2) / 2}\right)=\theta^{(j-i+2) / 2} \cdot[\iota(C)],
$$

so that $\theta^{(j-i+2) / 2} \cdot[\iota(C)]$ is in the image $\varphi_{*}\left(\mathscr{R}_{\bullet}\left(C^{[\infty]}\right)\right.$ ) (recall that $\left.\iota=\varphi_{1}\right)$. Moreover, we have shown in Proposition 6.3(ii) that $\mathscr{R}_{\bullet}\left(C^{[\infty]}\right)$ is stable under $[N]_{*}$ for all $N \geqslant 0$. This implies that the components $\theta^{(j-i+2) / 2} \cdot[\iota(C)]_{(j)}$ are also in the image $\varphi_{*}\left(\mathscr{R}_{\bullet}\left(C^{[\infty]}\right)\right)$. Since all generators of $\mathscr{T}_{\bullet}(J)$ are in $\varphi_{*}\left(\mathscr{R}_{\bullet}\left(C^{[\infty]}\right)\right)$, we obtain the inclusion $\mathscr{T}_{\bullet}(J) \subset \varphi_{*}\left(\mathscr{R}_{\bullet}\left(C^{[\infty]}\right)\right)$. 
To prove the other inclusion, observe that $\varphi_{*}\left(\mathscr{R}_{\bullet}\left(C^{[\infty]}\right)\right)$, being the union of the $\varphi_{n, *}\left(\mathscr{R}\left(C^{[n]}\right)\right)$ for $n \geqslant 0$, is also the union of the $\phi_{n, *}\left(\mathscr{R}\left(C^{n}\right)\right)$ for $n \geqslant 0$. Then it is enough to prove that $\phi_{n, *}\left(\mathscr{R}\left(C^{n}\right)\right) \subset \mathscr{T}(J)$ for all $n \geqslant 0$. This is done by an explicit calculation in terms of the generators of $\mathscr{R}\left(C^{n}\right)$.

As $\phi_{n, *}$ factors through $\phi_{n+1, *}$, we may assume $n \geqslant 2$. The ring $\mathscr{R}\left(C^{n}\right)$ is then generated by $\left\{\kappa_{i}\right\}, \psi,\left\{K_{j}\right\}$ and $\left\{\left[x_{0, j}\right]\right\}$, and $\left\{\left[\Delta_{k, l}\right]\right\}$. We make a change of variables

$$
\eta_{j}:=\frac{1}{2} K_{j}+\left[x_{0, j}\right]+\frac{1}{2} \psi,
$$

so that $\mathscr{R}\left(C^{n}\right)$ is also generated by $\left\{\kappa_{i}\right\}, \psi,\left\{\eta_{j}\right\}$ and $\left\{\left[x_{0, j}\right]\right\}$, and $\left\{\left[\Delta_{k, l}\right]\right\}$. Let $\alpha \in \mathscr{R}\left(C^{n}\right)$ be a monomial in those generators. We would like to show that $\phi_{n, *}(\alpha) \in \mathscr{T}(J)$.

A first step is to separate the variables $\left\{\kappa_{i}\right\}$ and $\psi$ from the rest. Write $\alpha=\beta \cdot \gamma$, with $\beta$ collecting all factors of $\left\{\kappa_{i}\right\}$ and $\psi$. Then $\beta$ is the pull-back of a class $\beta_{0} \in \mathscr{R}(S)$ via $p^{n}: C^{n} \rightarrow S$. Since $p^{n}=\pi \circ \phi_{n}$ (recall that $\pi$ is the map from $J$ to $S$ ), we find

$$
\phi_{n, *}(\alpha)=\phi_{n, *}\left(p^{n, *}\left(\beta_{0}\right) \cdot \gamma\right)=\phi_{n, *}\left(\phi_{n}^{*} \pi^{*}\left(\beta_{0}\right) \cdot \gamma\right)=\pi^{*}\left(\beta_{0}\right) \cdot \phi_{n, *}(\gamma) .
$$

Thanks to the isomorphisms (4.8), we have $\pi^{*}\left(\beta_{0}\right) \in \mathscr{T}(J)$. So it remains to prove that $\phi_{n, *}(\gamma) \in$ $\mathscr{T}(J)$, or in other words, we may assume that $\alpha$ is a monomial in $\left\{\eta_{j}\right\},\left\{\left[x_{0, j}\right]\right\}$ and $\left\{\left[\Delta_{k, l}\right]\right\}$ only.

A second step is to eliminate multiplicities in the variables $\left\{\left[\Delta_{k, l}\right]\right\}$. Consider for example $[\Delta]=\left[\Delta_{1,2}\right] \in \mathscr{R}\left(C^{2}\right)$. Denote by $\Delta: C \rightarrow C^{2}$ the diagonal map and by $\operatorname{pr}_{2}: C^{2} \rightarrow C$ the second projection. Then we have

$$
[\Delta]^{2}=\Delta_{*}\left(\Delta^{*}([\Delta])\right)=-\Delta_{*}(K)=-\Delta_{*}\left(\Delta^{*} \operatorname{pr}_{2}^{*}(K)\right)=-[\Delta] \cdot K_{2} .
$$

By pulling back to $C^{n}$, we obtain, for $1 \leqslant k<l \leqslant n$,

$$
\left[\Delta_{k, l}\right]^{2}=-\left[\Delta_{k, l}\right] \cdot K_{l}=-\left[\Delta_{k, l}\right] \cdot\left(2 \eta_{l}-2\left[x_{0, l}\right]-\psi\right) .
$$

Together with the first step, this allows us to reduce to the case where $\alpha$ is a monomial in $\left\{\eta_{j}\right\}$, $\left\{\left[x_{0, j}\right]\right\}$ and $\left\{\left[\Delta_{k, l}\right]\right\}$, with multiplicity at most 1 for each $\left[\Delta_{k, l}\right]$.

Further, we may permute the indices of the $\left\{\left[\Delta_{k, l}\right]\right\}$-factors by applying the identity

$$
\left[\Delta_{k, l}\right] \cdot\left[\Delta_{l, m}\right]=\left[\Delta_{k, m}\right] \cdot\left[\Delta_{l, m}\right] .
$$

More precisely, if $I=\left\{i_{1}, i_{2}, \ldots, i_{q}\right\}$ is a subset of $\{1, \ldots, n\}$, we define the symbol

$$
\left[\Delta_{I}\right]:=\left[\Delta_{i_{1}, i_{2}}\right] \cdot\left[\Delta_{i_{1}, i_{3}}\right] \cdots\left[\Delta_{i_{1}, i_{q}}\right] .
$$

It follows from (6.4) that $\left[\Delta_{I}\right]$ is well defined. Also, we have identities $\left[\Delta_{I}\right] \cdot \eta_{i_{1}}=\left[\Delta_{I}\right] \cdot \eta_{i_{2}}=$ $\cdots=\left[\Delta_{I}\right] \cdot \eta_{i_{q}}$ and $\left[\Delta_{I}\right] \cdot\left[x_{0, i_{1}}\right]=\left[\Delta_{I}\right] \cdot\left[x_{0, i_{2}}\right]=\cdots=\left[\Delta_{I}\right] \cdot\left[x_{0, i_{q}}\right]$. So for $r, s \geqslant 0$, we can write

$$
\left(\eta^{r}\left[x_{0}\right]^{s}\right)_{\Delta_{I}}:=\left[\Delta_{I}\right] \cdot \eta_{i_{1}}^{r} \cdot\left[x_{0, i_{1}}\right]^{s} .
$$

Combining with the first two steps, we may assume that the $\left\{\left[\Delta_{k, l}\right]\right\}$-factors of $\alpha$ take the form

$$
\left[\Delta_{I_{1}}\right] \cdot\left[\Delta_{I_{2}}\right] \cdots\left[\Delta_{I_{m}}\right]
$$

where the $I_{k}$ are subsets of $\{1,2, \ldots, n\}$ satisfying $I_{k} \cap I_{l}=\emptyset$ for $k \neq l$. This means that we are reduced to the case where $\alpha$ is of the form

$$
\alpha=\prod_{k \in\{1, \ldots, m\}}\left(\eta^{r_{k}}\left[x_{0}\right]^{s_{k}}\right)_{\Delta_{I_{k}}} \cdot \prod_{\substack{j \in\{1, \ldots, n\} \\ j \notin \cup_{k} I_{k}}}\left(\eta_{j}^{u_{j}}\left[x_{0, j}\right]^{v_{j}}\right),
$$




\section{A tale of TWO TAUTOLOGICAL Rings}

with $I_{1}, \ldots, I_{m}$ and $\{j\}$ pairwise disjoint. In this case, the calculation of $\phi_{n, *}(\alpha)$ is rather straightforward: it follows almost from the definitions of $[N]$ and $*$ that

$$
\phi_{n, *}(\alpha)=\prod_{k \in\{1, \ldots, m\}}^{*}\left[\# I_{k}\right]_{*} \iota_{*}\left(\eta^{r_{k}}\left[x_{0}\right]^{s_{k}}\right) * \prod_{\substack{j \in\{1, \ldots, n\} \\ j \notin \cup_{k} I_{k}}}^{*} \iota_{*}\left(\eta^{u_{j}}\left[x_{0}\right]^{v_{j}}\right),
$$

where $\Pi^{*}$ stands for product with respect to $*$, and $\# I_{k}$ the cardinality of $I_{k}$.

Now, since $\mathscr{T}(J)$ is stable under $[N]_{*}$ and $*$, the last step is to prove that $\iota_{*}\left(\eta^{r}\left[x_{0}\right]^{s}\right) \in \mathscr{T}(J)$ for all $r, s \geqslant 0$. By the identity $\eta=\iota^{*}(\theta)$, we have $\iota_{*}\left(\eta^{r}\left[x_{0}\right]^{s}\right)=\theta^{r} \cdot \iota_{*}\left(\left[x_{0}\right]^{s}\right)$, which further reduces the proof to showing that $\iota_{*}\left(\left[x_{0}\right]^{s}\right) \in \mathscr{T}(J)$. Note that $\iota_{*}\left(\left[x_{0}\right]^{0}\right)=[\iota(C)] \in \mathscr{T}(J)$ and $\iota_{*}\left(\left[x_{0}\right]\right)=[o] \in \mathscr{T}(J)$ (recall that $[o]$ is the class of the zero section). For $s \geqslant 2$, we have

$$
\iota_{*}\left(\left[x_{0}\right]^{s}\right)=\iota_{*} x_{0, *} x_{0}^{*}\left(\left[x_{0}\right]^{s-1}\right)=o_{*}\left((-\psi)^{s-1}\right) \in \mathscr{T}(J) .
$$

The proof of the inclusion $\varphi_{*}\left(\mathscr{R}_{\bullet}\left(C^{[\infty]}\right)\right) \subset \mathscr{T}_{\bullet}(J)$ is thus completed.

Proof of Theorem 6.4. By Proposition 6.3(i) and (iv), we know that $L \in \mathscr{R} \bullet\left(C^{[\infty]}\right)$ and that $\mathscr{R}_{\bullet}\left(C^{[\infty]}\right)$ is stable under $\xi \cap-$. Then the isomorphism (6.3) follows immediately from Lemma 6.5 and Proposition 6.6. By applying the Fourier transform $\mathscr{F}$ and Proposition 6.3(iii), we also obtain (6.2).

Remark 6.7. Previously, Moonen and Polishchuk considered much bigger tautological rings of $C^{[\infty]}$ and $J$, for which they obtained results similar to Theorem 6.4 [MP10, Corollary 8.6]. The advantage of our minimalist version is that one can use the $\mathfrak{s l}_{2}$-machinery on $\mathscr{T}(J)$ to study enumerative problems on $\mathscr{R}\left(C^{[n]}\right)$.

\section{Tautological relations and Gorenstein properties}

The $\mathfrak{s l}_{2}$-action on the Jacobian side provides relations between tautological classes. Using these relations, we study the Gorenstein property for $\mathscr{R}\left(\mathscr{M}_{g, 1}\right)$ (respectively, $\mathscr{R}\left(\mathscr{M}_{g}\right)$ ). We also formulate the corresponding Gorenstein property for the tautological ring $\mathscr{T}\left(\mathscr{J}_{g, 1}\right)$ of the universal Jacobian. Then, we prove that $\mathscr{T}\left(\mathscr{J}_{g, 1}\right)$ being Gorenstein is equivalent to $\mathscr{R}\left(\mathscr{C}_{g, 1}^{[n]}\right)$ being Gorenstein for all $n \geqslant 0$. A computation confirms the Gorenstein properties for small $g$, and suggests when these properties may not hold.

7.1. Relations via $\mathfrak{s l}_{2}$. We explain how the $\mathfrak{s l}_{2}$-action gives relations in $\mathscr{T}(J)$. Following the isomorphisms (4.8), we identify $\mathscr{R}(S)$ with $\oplus_{i=0}^{d} \mathscr{T}_{(0,2 i)}(J)$ via the map $\pi^{*}$. Then we obtain relations in $\mathscr{R}(S)$ by restriction.

The idea is due to Polishchuk [Pol05]. By Theorem 4.5, the space of polynomial relations between $\left\{p_{i, j}\right\}$ and $\psi$ is stable under the action of $\mathscr{D}$. In other words, if $P$ is a polynomial in $\left\{p_{i, j}\right\}$ and $\psi$, then $P\left(\left\{p_{i, j}\right\}, \psi\right)=0$ implies $\mathscr{D}\left(P\left(\left\{p_{i, j}\right\}, \psi\right)\right)=0$. Now, consider monomials

$$
\alpha=\psi^{s} p_{i_{1}, j_{1}}^{r_{1}} p_{i_{2}, j_{2}}^{r_{2}} \cdots p_{i_{m}, j_{m}}^{r_{m}} \quad \text { with } \quad I:=r_{1} i_{1}+r_{2} i_{2}+\cdots+r_{m} i_{m}>2 g .
$$

By definition $\alpha \in \oplus_{j} \mathrm{CH}_{(I, j)}(J)$. But since $I>2 g$, we know from the decomposition (3.7) that $\alpha=0$. In terms of the Dutch house, the class $\alpha$ is simply outside the house. It follows that we have relations

$$
\alpha=0, \quad \mathscr{D}(\alpha)=0, \quad \mathscr{D}^{2}(\alpha)=0, \quad \cdots
$$


7.2. This argument leads to the following formal definition. Let $i, j$ run through all integers such that $i \leqslant j+2$ and that $i+j$ is even. Define

$$
\mathscr{A}:=\mathbb{Q}\left[\left\{x_{i, j}\right\}, y\right] /\left\langle x_{0,0}-g,\left\{x_{i, j}\right\}_{i<0},\left\{x_{i, j}\right\}_{j<0},\left\{x_{i, j}\right\}_{j>2 g-2}\right\rangle .
$$

In other words, the ring $\mathscr{A}$ is a polynomial ring in variables $\left\{x_{i, j}\right\}$ and $y$, with the convention that $x_{0,0}=g$ and $x_{i, j}=0$ when $i<0$ or $j<0$ or $j>2 g-2$ (same as the classes $\left\{p_{i, j}\right\}$ ). We introduce a bigrading $\mathscr{A}=\oplus_{i, j} \mathscr{A}_{(i, j)}$ by the requirement that $x_{i, j} \in \mathscr{A}_{(i, j)}$ and $y \in \mathscr{A}_{(0,2)}$. Define operators $E, F$ and $H$ on $\mathscr{A}$ by

$$
\begin{array}{ll}
E: \mathscr{A}_{(i, j)} \rightarrow \mathscr{A}_{(i+2, j)}, & \alpha \mapsto x_{2,0} \cdot \alpha, \\
F: \mathscr{A}_{(i, j)} \rightarrow \mathscr{A}_{(i-2, j)}, & \alpha \mapsto F(\alpha), \\
H: \mathscr{A}_{(i, j)} \rightarrow \mathscr{A}_{(i, j)}, & \alpha \mapsto(i-g) \alpha,
\end{array}
$$

where

$$
F:=\frac{1}{2} \sum_{i, j, k, l}\left(y x_{i-1, j-1} x_{k-1, l-1}-\left(\begin{array}{c}
i+k-2 \\
i-1
\end{array}\right) x_{i+k-2, j+l}\right) \partial x_{i, j} \partial x_{k, l}+\sum_{i, j} x_{i-2, j} \partial x_{i, j} .
$$

It is not difficult to verify that these operators generate a $\mathbb{Q}$-linear representation $\mathfrak{s l}_{2} \rightarrow \operatorname{End}_{\mathbb{Q}}(\mathscr{A})$. Theorem 4.5 can then be reformulated as the existence of a surjective morphism of $\mathfrak{s l}_{2}$-representations $\mathscr{A} \rightarrow \mathscr{T}(J)$, which maps $x_{i, j}$ to $p_{i, j}$ and $y$ to $\psi$.

7.3. Denote by $\operatorname{Mon}_{(i, j)}$ the set of non-zero monomials in $\mathscr{A}_{(i, j)}$ (also without $x_{0,0}$ as a factor). For convenience we set $\operatorname{Mon}_{(0,0)}:=\{1\}$. Consider the quotient

$$
\tilde{\mathscr{T}}:=\mathscr{A} /\left\langle\left\{F^{\nu}\left(\operatorname{Mon}_{(i, j)}\right)\right\}_{i>2 g, \nu \geqslant 0}\right\rangle .
$$

The ring $\tilde{\mathscr{T}}$ inherits a bigrading $\tilde{\mathscr{T}}=\oplus_{i, j} \tilde{\mathscr{T}}_{(i, j)}$ from $\mathscr{A}$. The operators $E, F$ and $H$ induce operators on $\tilde{\mathscr{T}}$, which we denote by $e, f$ and $h$, respectively. Again, we obtain a representation $\mathfrak{s l}_{2} \rightarrow \operatorname{End}_{\mathbb{Q}}(\tilde{\mathscr{T}})$. Further, since $e^{g+1}=f^{g+1}=0$, we formally define the Fourier transform on $\tilde{\mathscr{T}}$ by

$$
\mathscr{F}:=\exp (e) \exp (-f) \exp (e) .
$$

We also define the subring $\tilde{\mathscr{R}}=\oplus_{i} \tilde{\mathscr{T}}_{(0,2 i)}$, graded by $\tilde{\mathscr{R}}=\oplus_{i} \tilde{\mathscr{R}}^{i}$ with $\tilde{\mathscr{R}}^{i}:=\tilde{\mathscr{T}}_{(0,2 i)}$. We have

$$
\tilde{\mathscr{R}}^{i}=\mathscr{A}_{(0,2 i)} /\left\langle\left\{F^{I}\left(\operatorname{Mon}_{(2 I, 2 i)}\right)\right\}_{I>g}\right\rangle=\mathscr{A}_{(0,2 i)} /\left\langle F^{g+1}\left(\operatorname{Mon}_{(2 g+2,2 i)}\right)\right\rangle .
$$

Figure 3 illustrates the construction of $\tilde{\mathscr{R}}$ : take monomials on the $(2 g+2)$ th column of the Dutch house (white blocks), and then apply the operator $F$ a total of $(g+1)$ times to obtain relations between the generators (black blocks).

To summarize this formal approach, we have the following proposition.

Proposition 7.4. There are surjective maps

$$
\Theta: \tilde{\mathscr{T}} \rightarrow \mathscr{T}(J) \text { and }\left.\quad \Theta\right|_{\tilde{\mathscr{R}}}: \tilde{\mathscr{R}} \rightarrow \mathscr{R}(S),
$$

which map $x_{i, j}$ to $p_{i, j}$ and $y$ to $\psi$, respectively.

We look at $\tilde{\mathscr{R}}$ in more detail. The following lemma shows that one can eliminate certain monomials that produce trivial relations.

Lemma 7.5. For all $\alpha \in \operatorname{Mon}_{(2 g+2,2 i)}$ of the form $\alpha=x_{2,0} \cdot \beta$, we have $F^{g+1}(\alpha)=0$. 


\section{A TALE OF TWO TAUTOLOGICAL RINGS}

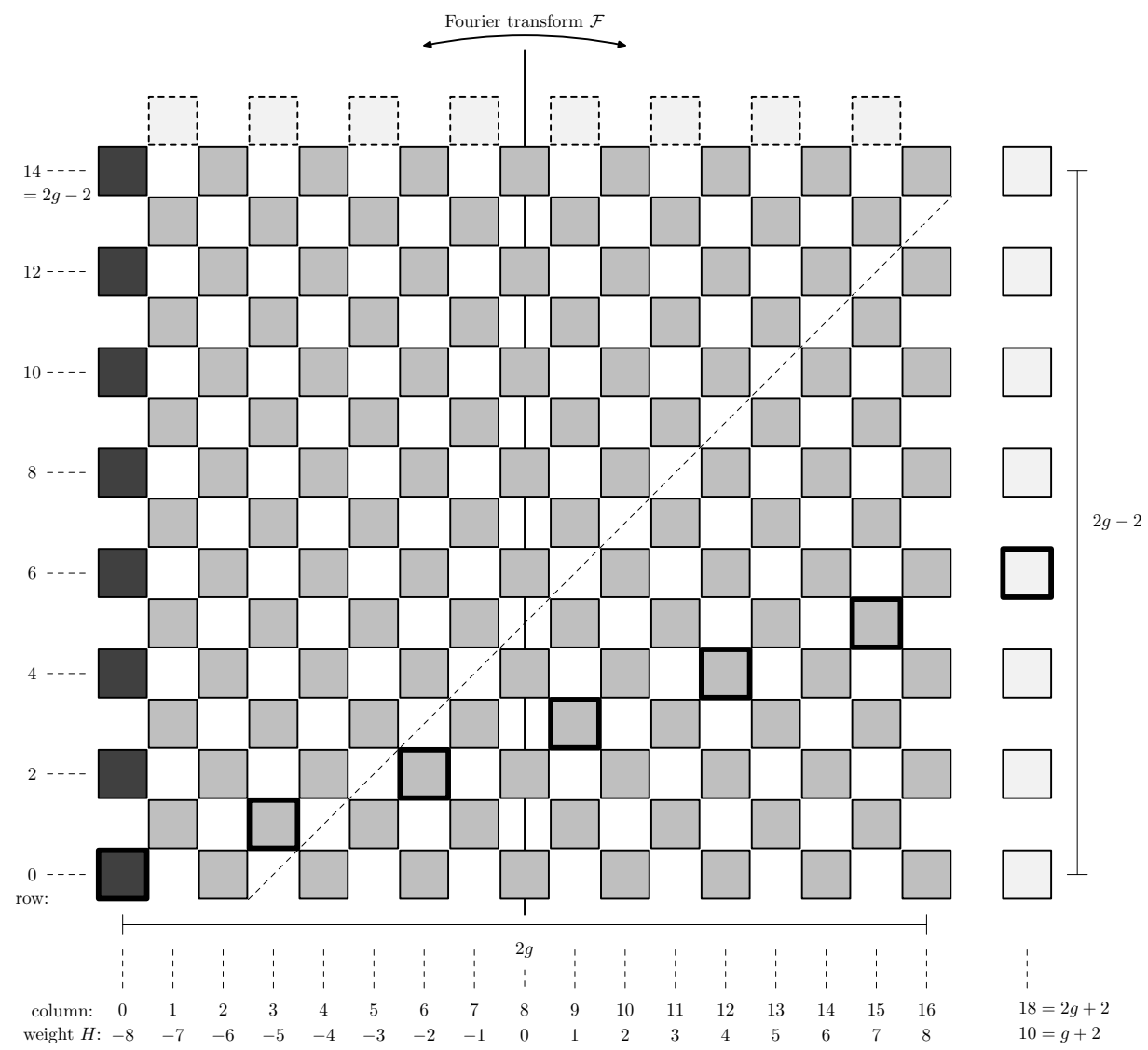

FiguRE 3. Producing relations $(g=8)$

Proof. We know that $F^{g+1}(\alpha)=F^{g+1}\left(x_{2,0} \cdot \beta\right)=F^{g+1} E(\beta)$. Using a combinatorial identity for $\mathfrak{s l}_{2}$-representations (see for example [Moo09, Lemma 2.4]), we find

$$
F^{g+1} E(\beta)=E F^{g+1}(\beta)-(g+1)(g+1-g-1+1-1) F^{g}(\beta)=E F^{g+1}(\beta) .
$$

On the other hand $F^{g+1}(\beta) \in \mathscr{A}_{(-2,2 i)}=0$, which implies $F^{g+1}(\alpha)=0$.

As a result, if we write $\operatorname{mon}_{(2 g+2,2 i)} \subset \operatorname{Mon}_{(2 g+2,2 i)}$ for the subset of monomials without $x_{2,0}$ as a factor, then we have

$$
\tilde{\mathscr{R}}^{i}=\mathscr{A}_{(0,2 i)} /\left\langle F^{g+1}\left(\operatorname{mon}_{(2 g+2,2 i)}\right)\right\rangle .
$$

The bold blocks in Figure 3 describe the lower bound of $i$ such that $\operatorname{mon}_{(2 g+2,2 i)}$ is non-empty. In numerical terms, we have $x_{3,1}^{2 i} \in \mathscr{A}_{(6 i, 2 i)}$ and $6 i>2 g$ means $i>g / 3$. Hence $\operatorname{mon}_{(2 g+2,2 i)}=\emptyset$ for all $i \leqslant\lfloor g / 3\rfloor$.

Proposition 7.6. The elements $x_{0,2}, \ldots, x_{0,2\lfloor g / 3\rfloor}$ and $y$ generate $\tilde{\mathscr{R}}$, with no relations in $\tilde{\mathscr{R}}^{i}$ for $i \leqslant\lfloor g / 3\rfloor$.

Together with (4.7), this gives a new proof of Ionel's result [Ion05, Theorem 1.5] that $\mathscr{R}(S)$ is generated by $\kappa_{1}, \ldots, \kappa_{\lfloor g / 3\rfloor}$ and $\psi$.

Proof. The second part is immediate from (7.3) and the fact that $\operatorname{mon}_{(2 g+2,2 i)}=\emptyset$ for all $i \leqslant$ $\lfloor g / 3\rfloor$. For the first part, the goal is to relate all $x_{0,2 i}$ with $i>g / 3$ to the elements $x_{0,2}, \ldots, x_{0,2\lfloor g / 3\rfloor}$ 
and $y$, and the idea is to use specific monomials to get these relations.

We proceed by induction. Suppose all $\left\{x_{0,2 j}\right\}_{g / 3<j<i}$ can be expressed in terms of the elements $x_{0,2}, \ldots, x_{0,2\lfloor g / 3\rfloor}$ and $y$. Then consider the monomial $x_{3,1}^{2 i} \in \mathscr{A}_{(6 i, 2 i)}$. Applying the operator $F$ a total of $3 i$ times, we get $F^{3 i}\left(x_{3,1}^{2 i}\right)$, which is an element of $\mathscr{A}_{(0,2 i)}$ and vanishes in $\tilde{\mathscr{R}}$. On the other hand, by going through the definition of $F$ we find

$$
F^{3 i}\left(x_{3,1}^{2 i}\right)=c x_{0,2 i}+\alpha,
$$

where $\alpha$ is a polynomial in $\left\{x_{0,2 j}\right\}_{j<i}$ and $y$. It remains to show that $c$ is non-zero.

The observation is the following: when we apply the operator $F$, the minus sign occurs every time two factors $\left(x_{i, j}, x_{k, l}\right)$ are merged into $x_{i+k-2, j+l}$. If we start from $x_{3,1}^{2 i}$ and arrive at $x_{0,2 i}$, no matter how we proceed we have to do the merging $(2 i-1)$ times. This means that all non-zero summands of $c$ are of the form $(-1)^{2 g-1}$ times a positive number, hence negative. Then the sum $c$ is also negative.

7.7. Computing $\mathscr{R}\left(\mathscr{M}_{\boldsymbol{g}, \mathbf{1}}\right)$. Our colleague Li Ma made a $\mathrm{C}++$ program that computes $\tilde{\mathscr{R}}$ for a given genus $g$. It calculates relations and outputs the dimension of each component $\tilde{\mathscr{R}}^{i}$.

Meanwhile, based on an algorithm developed by Liu and Xu [LX12], Bergvall computed the intersection numbers in $\mathscr{R}\left(\mathscr{M}_{g, 1}\right)$ for many values of $g$ (see [Ber11, Section 4.2]). His computation then gives the dimensions of the Gorenstein quotient $\mathscr{G}\left(\mathscr{M}_{g, 1}\right)$, which is the quotient of $\mathscr{R}\left(\mathscr{M}_{g, 1}\right)$ by those classes that pair zero with all opposite degree classes. Note that this computation is formal and does not involve actual relations in $\mathscr{R}\left(\mathscr{M}_{g, 1}\right)$.

There are surjective maps $\tilde{\mathscr{R}} \rightarrow \mathscr{R}\left(\mathscr{M}_{g, 1}\right) \rightarrow \mathscr{G}\left(\mathscr{M}_{g, 1}\right)$. Our computation shows that for $g \leqslant 19$, the dimensions of $\tilde{\mathscr{R}}$ and $\mathscr{G}\left(\mathscr{M}_{g, 1}\right)$ are equal, which means that we have $\tilde{\mathscr{R}} \simeq \mathscr{R}\left(\mathscr{M}_{g, 1}\right) \simeq$ $\mathscr{G}\left(\mathscr{M}_{g, 1}\right)$. In particular, we can confirm the following (for $g \leqslant 9$ this has been obtained independently by Bergvall; see [Ber11, Section 4.4]).

Corollary 7.8. The ring $\mathscr{R}\left(\mathscr{M}_{g, 1}\right)$ is Gorenstein for $g \leqslant 19$.

However, the computer output is negative for $g=20$ and some greater values of $g$. There, the dimensions of $\tilde{\mathscr{R}}$ are simply not symmetric. Again by comparing with the dimensions of $\mathscr{G}\left(\mathscr{M}_{g, 1}\right)$, we know exactly how many relations are missing. The numbers are listed in Table 1 . Note that for $g \geqslant 25$, we only calculated a range near the middle degree, presuming that $\mathscr{R}\left(\mathscr{M}_{g, 1}\right)$ behaves well near the top.

7.9. We include a brief discussion about $\mathscr{R}\left(\mathscr{M}_{g}\right)$. Recall that $\mathscr{R}\left(\mathscr{M}_{g}\right)$ is the $\mathbb{Q}$-subalgebra of $\mathrm{CH}\left(\mathscr{M}_{g}\right)$ generated by $\left\{\kappa_{i}\right\}$. Faber's original conjecture predicts that $\mathscr{R}\left(\mathscr{M}_{g}\right)$ is Gorenstein with socle in degree $g-2$ [Fab99, Conjecture 1].

Denote by $q: \mathscr{M}_{g, 1} \rightarrow \mathscr{M}_{g}$ the forgetful map. It is not difficult to verify that $q_{*}\left(\mathscr{R}\left(\mathscr{M}_{g, 1}\right)\right)=$ $\mathscr{R}\left(\mathscr{M}_{g}\right)$. More precisely, for $\psi^{s} \kappa_{i_{1}}^{r_{1}} \cdots \kappa_{i_{m}}^{r_{m}} \in \mathscr{R}\left(\mathscr{M}_{g, 1}\right)$, we have

$$
q_{*}\left(\psi^{s} \kappa_{i_{1}}^{r_{1}} \cdots \kappa_{i_{m}}^{r_{m}}\right)=\kappa_{s-1} \kappa_{i_{1}}^{r_{1}} \cdots \kappa_{i_{m}}^{r_{m}},
$$

with the convention that $\kappa_{-1}=0$.

Together with (4.7), the identity above allows us to push relations in $\mathscr{R}\left(\mathscr{M}_{g, 1}\right)$ forward to $\mathscr{R}\left(\mathscr{M}_{g}\right)$. We used another computer program to do the work. Then we recover the well-known result of Faber and Zagier that $\mathscr{R}\left(\mathscr{M}_{g}\right)$ is Gorenstein for $g \leqslant 23$. Note that when $20 \leqslant g \leqslant 23$, the missing relations in $\mathscr{R}\left(\mathscr{M}_{g, 1}\right)$ do not affect the Gorenstein property for $\mathscr{R}\left(\mathscr{M}_{g}\right)$. 
A tAle of tWo tAUtological Rings

TABLE 1. Computer output for $g \leqslant 28$

\begin{tabular}{c|c|c}
\hline$g$ & $\mathscr{M}_{g, 1}$ & $\mathscr{M}_{g}$ \\
\hline$\leqslant 19$ & OK & OK \\
20 & codim 10: 1 missing & OK \\
21 & codim 11: 1 missing & OK \\
22 & codim 11: 1 missing & OK \\
23 & codim 12: 3 missing & OK \\
24 & codim 13: 2 missing & codim 12:1 missing \\
& codim 12: 4 missing & \\
25 & codim 13:5 missing & codim 12:1 missing \\
& codim 12:1 missing & \\
26 & codim 14: 6 missing & codim 13: 1 missing \\
& codim 13: 6 missing & \\
27 & codim 15: 3 missing & codim 14: 1 missing \\
& codim 14: 11 missing & codim 13: 1 missing \\
& codim 13: 1 missing & \\
\hline \multirow{2}{*}{28} & codim 15: 10 missing & codim 14: 2 missing \\
& codim 14: 10 missing & \\
\hline
\end{tabular}

From $g=24$ on, the computer output is again negative; see Table 1. Our computation for $g \leqslant 28$ suggests that we obtain exactly the same set of relations as the Faber-Zagier relations (see [PP13, Section 0.2]). Notably in the crucial case of $g=24$, we have not found the missing relation in codimension 12 . It is not known whether in theory we obtain the same relations.

7.10. We continue to study the Gorenstein properties for $\mathscr{R}\left(\mathscr{C}_{g, 1}^{[n]}\right)$. A key step is to establish a similar property for the Jacobian side. Denote by $\mathscr{J}_{g, 1}$ the universal Jacobian over $\mathscr{M}_{g, 1}$. The tautological ring $\mathscr{T}\left(\mathscr{J}_{g, 1}\right)$ is thus defined.

The following lemma locates the expected socle for $\mathscr{T}\left(\mathscr{J}_{g, 1}\right)$.

Lemma 7.11. We have $\mathscr{T}^{i}\left(\mathscr{J}_{g, 1}\right)=0$ for $i>2 g-1$ and

$$
\mathscr{T}^{2 g-1}\left(\mathscr{J}_{g, 1}\right)=\mathscr{T}_{(2 g-2)}^{2 g-1}\left(\mathscr{J}_{g, 1}\right) \simeq \mathbb{Q} .
$$

Proof. The surjective map $\phi_{g}: \mathscr{C}_{g, 1}^{g} \rightarrow \mathscr{J}_{g, 1}$ induces $\phi_{g}^{*}: \mathrm{CH}\left(\mathscr{J}_{g, 1}\right) \hookrightarrow \mathrm{CH}\left(\mathscr{C}_{g, 1}^{g}\right)$ which, by (6.2), restricts to an injective map $\phi_{g}^{*}: \mathscr{T}\left(\mathscr{J}_{g, 1}\right) \hookrightarrow \mathscr{R}\left(\mathscr{C}_{g, 1}^{g}\right)$. Then it follows from $(2.1)$ that $\mathscr{T}^{i}\left(\mathscr{J}_{g, 1}\right)=$ 0 for $i>2 g-1$, and that $\mathscr{T}^{2 g-1}\left(\mathscr{J}_{g, 1}\right)$ is at most 1-dimensional.

Recall from (4.8) that $\mathscr{T}_{(2 g-2)}^{g-1}\left(\mathscr{J}_{g, 1}\right) \simeq \mathscr{R}^{g-1}\left(\mathscr{M}_{g, 1}\right) \simeq \mathbb{Q}$. Applying the Fourier transform, we obtain

$$
\mathscr{T}_{(2 g-2)}^{2 g-1}\left(\mathscr{J}_{g, 1}\right)=\mathscr{F}\left(\mathscr{T}_{(2 g-2)}^{g-1}\left(\mathscr{J}_{g, 1}\right)\right) \simeq \mathbb{Q} .
$$

So $\mathscr{T}^{2 g-1}\left(\mathscr{J}_{g, 1}\right)$ is indeed 1-dimensional, and is concentrated in $\mathscr{T}_{(2 g-2)}^{2 g-1}\left(\mathscr{J}_{g, 1}\right)$.

With the socle condition verified, we consider, for $0 \leqslant i \leqslant 2 g-1$, the pairing

$$
\mathscr{T}^{i}\left(\mathscr{J}_{g, 1}\right) \times \mathscr{T}^{2 g-1-i}\left(\mathscr{J}_{g, 1}\right) \dot{\rightarrow} \mathscr{T}^{2 g-1}\left(\mathscr{J}_{g, 1}\right) \simeq \mathbb{Q} .
$$

There is the following analog of Speculation 2.8. 


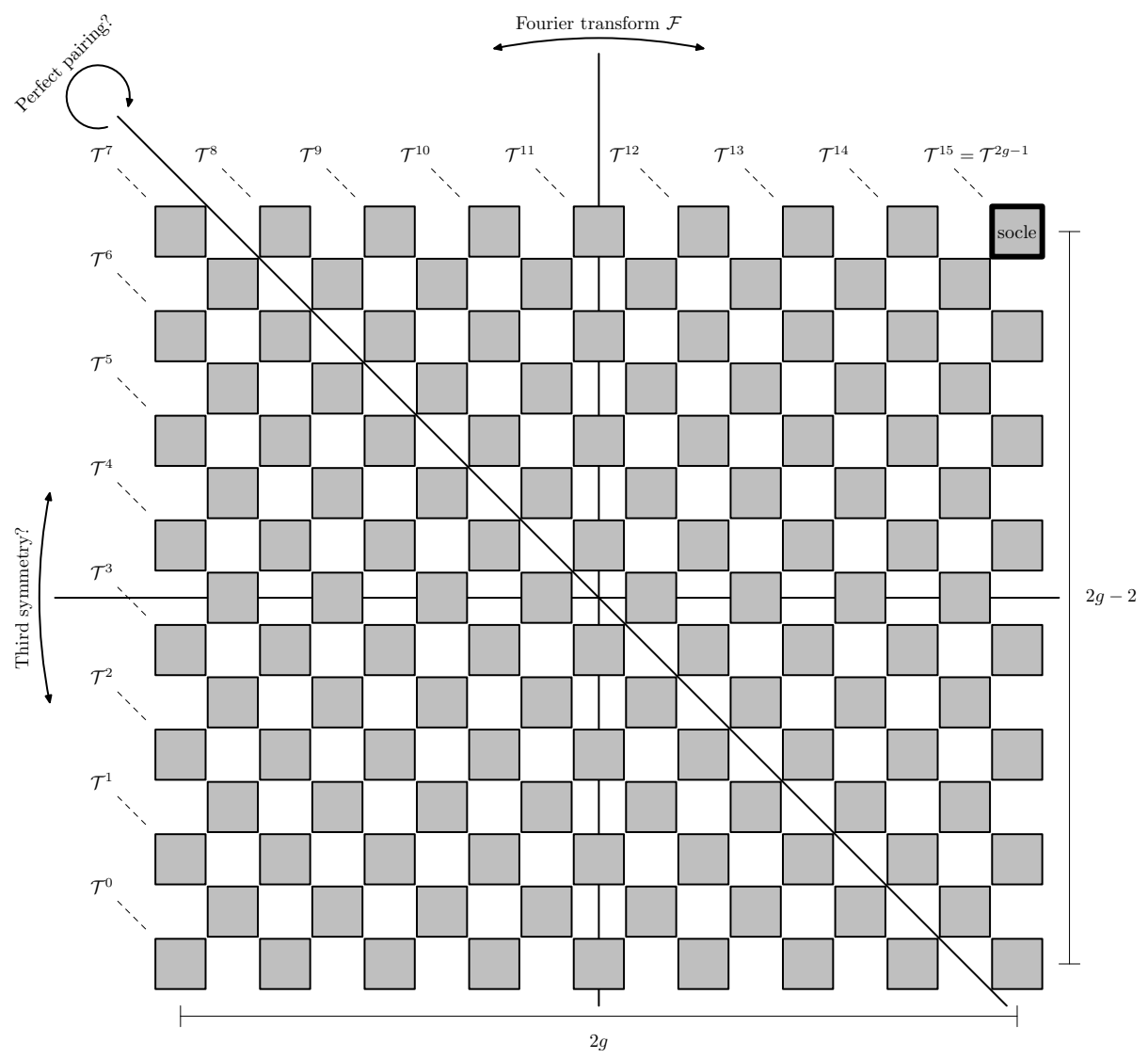

FiguRE 4. Pairings in $\mathscr{T}\left(\mathscr{J}_{g, 1}\right)(g=8)$

Speculation 7.12. For $0 \leqslant i \leqslant 2 g-1$, the pairing (7.4) is perfect. In other words, the ring $\mathscr{T}\left(\mathscr{J}_{g, 1}\right)$ is Gorenstein with socle in degree $2 g-1$.

The Dutch house gives an nice interpretation. In Figure 4, the socle component is located precisely in the upper right corner. Assuming the Gorenstein property, one would expect a rotational symmetry about the center of the picture. Together with the reflection symmetry about the middle vertical line (given by the Fourier transform), it would then imply a mysterious reflection symmetry about the middle horizontal line. In particular, one should have $\mathscr{T}_{(j)}\left(\mathscr{J}_{g, 1}\right)=0$ for $j>2 g-2$, and using the grading in $(3.7)$, a one-to-one correspondence between $\mathscr{T}_{(i, j)}\left(\mathscr{J}_{g, 1}\right)$ and $\mathscr{T}_{(i, 2 g-2-j)}\left(\mathscr{J}_{g, 1}\right)$.

7.13. Computing $\mathscr{T}(\mathscr{J} \boldsymbol{g}, \mathbf{1})$. Once again with the help of Li Ma, we computed the ring $\tilde{\mathscr{T}}$ defined in (7.1) and its pairings. The computer output shows that for $g \leqslant 7$, we do have $\tilde{\mathscr{T}}^{2 g-1} \simeq$ $\mathbb{Q}$ and perfect pairings between $\tilde{\mathscr{T}}^{i}$ and $\tilde{\mathscr{T}}^{2 g-1-i}$. Then, since the surjective map $\tilde{\mathscr{T}} \rightarrow \mathscr{T}\left(\mathscr{J}_{g, 1}\right)$ is an isomorphism at the socle level, it is in fact an isomorphism. In particular, we can confirm the following.

Corollary 7.14. The ring $\mathscr{T}\left(\mathscr{J}_{g, 1}\right)$ is Gorenstein for $g \leqslant 7$.

For $g=8$ and some greater values of $g$, however, the relations we find are not sufficient to deduce the Gorenstein property for $\mathscr{T}\left(\mathscr{J}_{g, 1}\right)$. 


\section{A tale of TWO TAUTOLOGICAL Rings}

Further, one of the main results of this section is an equivalence of Gorenstein properties.

Theorem 7.15. Fix $g>0$. The following three statements are equivalent:

(i) The ring $\mathscr{T}\left(\mathscr{J}_{g, 1}\right)$ is Gorenstein.

(ii) The ring $\mathscr{R}\left(\mathscr{C}_{g, 1}^{[n]}\right)$ is Gorenstein for some $n \geqslant 2 g-1$.

(iii) The ring $\mathscr{R}\left(\mathscr{C}_{g, 1}^{[n]}\right)$ is Gorenstein for all $n \geqslant 0$.

Proof. First assume $n \geqslant 2 g-1$. Recall that $\mathscr{C}_{g, 1}^{[n]}$ is a $\mathbb{P}^{n-g}$-bundle over $\mathscr{J}_{g, 1}$. Then we obtain from (5.4) and (6.2) an isomorphism of $\mathbb{Q}$-algebras

$$
\mathscr{R}\left(\mathscr{C}_{g, 1}^{[n]}\right) \simeq \mathscr{T}\left(\mathscr{J}_{g, 1}\right)\left[\xi_{n}\right] /\left\langle P\left(\xi_{n}\right)\right\rangle,
$$

where $P\left(\xi_{n}\right)$ is a polynomial in $\xi_{n}$ of degree $n-g+1$ with coefficients in $\mathscr{T}\left(\mathscr{J}_{g, 1}\right)$ (one shows by induction that the coefficients of $P\left(\xi_{n}\right)$ are in $\varphi_{n, *}\left(\mathscr{R}\left(\mathscr{C}_{g, 1}^{[n]}\right)\right)$, which by Proposition 6.6 equals $\left.\mathscr{T}\left(\mathscr{J}_{g, 1}\right)\right)$. In particular, we have for the socle components

$$
\mathscr{R}^{g-1+n}\left(\mathscr{C}_{g, 1}^{[n]}\right) \simeq \mathscr{T}^{2 g-1}\left(\mathscr{J}_{g, 1}\right) \cdot \xi_{n}^{n-g} \simeq \mathbb{Q} .
$$

For $0 \leqslant i \leqslant g-1+n$, we write $\mathscr{R}^{i}\left(\mathscr{C}_{g, 1}^{[n]}\right) \simeq \oplus_{j} \mathscr{T}^{i-j}\left(\mathscr{J}_{g, 1}\right) \cdot \xi_{n}^{j}$ with $\max \{0, i-2 g+1\} \leqslant j \leqslant$ $\min \{i, n-g\}$. Then the pairing (2.3) corresponds to

$$
\left(\bigoplus_{j} \mathscr{T}^{i-j}\left(\mathscr{J}_{g, 1}\right) \cdot \xi_{n}^{j}\right) \times\left(\bigoplus_{k} \mathscr{T}^{g-1+n-i-k}\left(\mathscr{J}_{g, 1}\right) \cdot \xi_{n}^{k}\right) \dot{\rightarrow} \mathscr{T}^{2 g-1}\left(\mathscr{J}_{g, 1}\right) \cdot \xi_{n}^{n-g} \simeq \mathbb{Q} .
$$

On the other hand, observe that

$$
\left(\mathscr{T}^{i-j}\left(\mathscr{J}_{g, 1}\right) \cdot \xi_{n}^{j}\right) \cdot\left(\mathscr{T}^{g-1+n-i-k}\left(\mathscr{J}_{g, 1}\right) \cdot \xi_{n}^{k}\right)=0 \quad \text { if } j+k<n-g .
$$

In other words, if we choose a suitable basis for each $\mathscr{R}^{i}\left(\mathscr{C}_{g, 1}^{[n]}\right)$, then the pairing matrix of (2.3) is block triangular. Moreover, the blocks on the diagonal correspond exactly to the case $j+k=n-g$, that is, to the pairing

$$
\left(\mathscr{T}^{i-j}\left(\mathscr{J}_{g, 1}\right) \cdot \xi_{n}^{j}\right) \times\left(\mathscr{T}^{2 g-1-i+j}\left(\mathscr{J}_{g, 1}\right) \cdot \xi_{n}^{n-g-j}\right) \dot{\rightarrow} \mathscr{T}^{2 g-1}\left(\mathscr{J}_{g, 1}\right) \cdot \xi_{n}^{n-g} \simeq \mathbb{Q},
$$

which in turn corresponds to the pairing

$$
\mathscr{T}^{i-j}\left(\mathscr{J}_{g, 1}\right) \times \mathscr{T}^{2 g-1-i+j}\left(\mathscr{J}_{g, 1}\right) \dot{\rightarrow} \mathscr{T}^{2 g-1}\left(\mathscr{J}_{g, 1}\right) \simeq \mathbb{Q} .
$$

In summary, saying that (2.3) is perfect for all $0 \leqslant i \leqslant g-1+n$ is equivalent to saying that (7.4) is perfect for all $0 \leqslant i \leqslant 2 g-1$. This settles the proof for (some or all) $n \geqslant 2 g-1$.

For the remaining $n$, the observation is that $\mathscr{R}\left(\mathscr{C}_{g, 1}^{[n]}\right)$ being Gorenstein implies $\mathscr{R}\left(\mathscr{C}_{g, 1}^{[n-1]}\right)$ being Gorenstein. To see this, take a class $\alpha \in \mathscr{R}^{i}\left(\mathscr{C}_{q, 1}^{[n-1]}\right)$ that pairs zero with all classes in $\mathscr{R}^{g+n-2-i}\left(\mathscr{C}_{g, 1}^{[n-1]}\right)$. Recall the correspondence $\delta_{n}: \mathscr{C}_{g, 1}^{[n]} \vdash \mathscr{C}_{g, 1}^{[n-1]}$ defined in (5.2), and consider the pairing of $\delta_{n}^{*}(\alpha)$ with all $\gamma \in \mathscr{R}^{g+n-1-i}\left(\mathscr{C}_{g, 1}^{[n]}\right)$. It then follows from the projection formula that each summand of $\delta_{n}^{*}(\alpha) \cdot \gamma$ is zero, and hence $\delta_{n}^{*}(\alpha) \cdot \gamma=0$. Assuming that $\mathscr{R}\left(\mathscr{C}_{g, 1}^{[n]}\right)$ is Gorenstein, we get $\delta_{n}^{*}(\alpha)=0$. Since $\delta_{n}^{*}$ is injective, we have $\alpha=0$ and $\mathscr{R}\left(\mathscr{C}_{g, 1}^{[n-1]}\right)$ is Gorenstein. The proof is thus completed.

By Corollary 7.14 and the discussion in Section 2, we obtain an immediate consequence. COROLlary 7.16. The rings $\mathscr{R}\left(\mathscr{C}_{g, 1}^{[n]}\right)$ and $\mathscr{R}\left(\mathscr{C}_{g}^{[n]}\right)$ are Gorenstein for $g \leqslant 7$ and for all $n \geqslant 0$. 
Finally, we conclude by proposing an alternative description of the tautological rings. As is explained in the introduction, this new description is coherent with Polishchuk's philosophy in [Pol05], and appears more geometric than the Gorenstein expectation.

ConjeCture 7.17. The map $\Theta: \tilde{\mathscr{T}} \rightarrow \mathscr{T}\left(\mathscr{J}_{g, 1}\right)$ in $(7.2)$ is an isomorphism.

\section{ACKNOWLEDGEMENTS}

This note is part of the author's PhD thesis, written during his stays at the Université Paris VI, the University of Amsterdam and the Radboud University Nijmegen. It also expands an earlier preprint [Yin12].

The author is deeply indebted to his thesis advisor Ben Moonen, who introduced him to the subject and encouraged him throughout this project. He is grateful to Li Ma, whose brilliant programming made this work much more meaningful. He also thanks Arnaud Beauville, Olof Bergvall, Alessandro Chiodo, Carel Faber, Gavril Farkas, Gerard van der Geer, Samuel Grushevsky, Felix Janda, Kefeng Liu, Rahul Pandharipande, Dan Petersen, Aaron Pixton, Sergey Shadrin, Mehdi Tavakol, Orsola Tommasi, Ravi Vakil, Claire Voisin (his co-advisor), Dmitry Zakharov and Shengmao Zhu for their valuable feedback and many useful discussions. The whole project was inspired by Robin de Jong's talk at the PCMI in the summer of 2011.

The computation was carried out on the Lisa compute cluster at SURFsara (available at http://surfsara.nl). The program code is available from the author upon request.

\section{REFERENCES}

Bea86 A. Beauville, Sur l'anneau de Chow d'une variété abélienne, Math. Ann. 273 (1986), no. 4, 647-651; http://dx.doi.org/10.1007/BF01472135.

Bea04 Algebraic cycles on Jacobian varieties, Compos. Math. 140 (2004), no. 3, 683-688; http://dx.doi.org/10.1112/S0010437X03000733.

Bea10 _ The action of $\mathrm{SL}_{2}$ on abelian varieties, J. Ramanujan Math. Soc. 25 (2010), no. 3, 253-263.

Ber11 O. Bergvall, Relations in the tautological ring of the universal curve, Master thesis, KTH Stockholm, 2011, available at http://www.su.se/polopoly_fs/1.228813.1426599391! /menu/standard/file/Rapport.pdf

DM91 C. Deninger and J. Murre, Motivic decomposition of abelian schemes and the Fourier transform, J. reine angew. Math. 422 (1991), 201-219; http://dx.doi.org/10.1515/crll.1991.422. 201.

Fab97 C. Faber, A non-vanishing result for the tautological ring of $\mathcal{M}_{g}$, arXiv:math.AG/9711219.

Fab99_A A conjectural description of the tautological ring of the moduli space of curves, in Moduli of Curves and Abelian Varieties, Aspects Math., vol. E33 (Vieweg, Braunschweig, 1999), 109129.

Fab13 , Tautological algebras of moduli spaces of curves, in Moduli spaces of Riemann surfaces, IAS/Park City Math. Ser., vol. 20 (Amer. Math. Soc., Providence, RI, 2013), 197-219.

FP00 C. Faber and R. Pandharipande, Logarithmic series and Hodge integrals in the tautological ring (with an appendix by Don Zagier), Michigan Math. J. 48 (2000), 215-252; http://dx.doi. org/10.1307/mmj/1030132716.

FP05_L Relative maps and tautological classes, J. Eur. Math. Soc. (JEMS) 7 (2005), no. 1, 13-49; http://dx.doi.org/10.4171/JEMS/20. 


\section{A tale of TWO TAUTOLOGICAL Rings}

FP13 Tautological and non-tautological cohomology of the moduli space of curves, in Handbook of Moduli. Vol. I, Adv. Lect. Math. (ALM), vol. 24 (Int. Press, Somerville, MA, 2013), 293-330.

vdGK07 G. van der Geer and A. Kouvidakis, Cycle relations on Jacobian varieties (with an appendix by Don Zagier), Compos. Math. 143 (2007), no. 4, 900-908; http://dx.doi.org/10.1112/ S0010437X07002849.

Her07 F. Herbaut, Algebraic cycles on the Jacobian of a curve with a linear system of given dimension, Compos. Math. 143 (2007), no. 4, 883-899; http://dx.doi.org/10.1112/ S0010437X07002850.

Ion05 E. N. Ionel, Relations in the tautological ring of $\mathcal{M}_{g}$, Duke Math. J. 129 (2005), no. 1, 157-186; http://dx.doi.org/10.1215/S0012-7094-04-12916-1.

Jan13 F. Janda, Tautological relations in moduli spaces of weighted pointed curves, arXiv:1306.6580.

Kün93 K. Künnemann, A Lefschetz decomposition for Chow motives of abelian schemes, Invent. Math. 113 (1993), no. 1, 85-102; http://dx.doi.org/10.1007/BF01244303.

KV96 S.-I. Kimura and A. Vistoli, Chow rings of infinite symmetric products, Duke Math. J. 85 (1996), no. 2, 411-430; http://dx.doi.org/10.1215/S0012-7094-96-08517-8.

Loo95 E. Looijenga, On the tautological ring of $\mathcal{M}_{g}$, Invent. Math. 121 (1995), no. 2, 411-419; http: //dx.doi.org/10.1007/BF01884306.

LX12 K. Liu and H. Xu, Computing top intersections in the tautological ring of $\mathcal{M}_{g}$, Math. Z. 270 (2012), no. 3-4, 819-837; http://dx.doi.org/10.1007/s00209-010-0828-9.

Moo09 B. Moonen, Relations between tautological cycles on Jacobians, Comment. Math. Helv. 84 (2009), no. 3, 471-502; http://dx.doi.org/10.4171/CMH/170.

MP10 B. Moonen and A. Polishchuk, Algebraic cycles on the relative symmetric powers and on the relative Jacobian of a family of curves. II, J. Inst. Math. Jussieu 9 (2010), no. 4, 799-846; http://dx.doi.org/10.1017/S147474801000006X.

Mum83 D. Mumford, Towards an enumerative geometry of the moduli space of curves, in Arithmetic and Geometry, Vol. II, Progr. Math., vol. 36 (Birkhäuser Boston, Boston, MA, 1983), 271-328; http://dx.doi.org/10.1007/978-1-4757-9286-7_12.

Pan02 R. Pandharipande, Three questions in Gromov-Witten theory, Proceedings of the International Congress of Mathematicians, Vol. II (Beijing, 2002) (Higher Ed. Press, Beijing, 2002), 503-512.

Pet13 D. Petersen, The tautological ring of the space of pointed genus two curves of compact type, arXiv:1310.7369.

Pet15_, Poincaré duality of wonderful compactifications and tautological rings, Int. Math. Res. Not., to appear, arXiv:1501.04742.

Pix12 A. Pixton, Conjectural relations in the tautological ring of $\overline{\mathcal{M}}_{g, n}$, arXiv:1207.1918.

Pix13 - The tautological ring of the moduli space of curves, $\mathrm{PhD}$ thesis, Princeton University, 2013, available at http://math.mit.edu/ apixton/papers/tautological.pdf.

Pol05 A. Polishchuk, Universal algebraic equivalences between tautological cycles on Jacobians of curves, Math. Z. 251 (2005), no. 4, 875-897; http://dx.doi.org/10.1007/ s00209-005-0838-1.

Pol07a , Algebraic cycles on the relative symmetric powers and on the relative Jacobian of a family of curves. I, Selecta Math. (N.S.) 13 (2007), no. 3, 531-569; http://dx.doi.org/10. 1007/s00029-008-0049-9.

Pol07b L Lie symmetries of the Chow group of a Jacobian and the tautological subring, J. Algebraic Geom. 16 (2007), no. 3, 459-476; http://dx.doi.org/10.1090/ S1056-3911-06-00431-0.

Pol08 - Fourier-stable subrings in the Chow rings of abelian varieties, Math. Res. Lett. 15 (2008), no. 4, 705-714; http://dx.doi.org/10.4310/MRL.2008.v15.n4.a9.

PP13 R. Pandharipande and A. Pixton, Relations in the tautological ring of the moduli space of curves, arXiv:1301.4561. 


\section{Q. YIN}

PPZ15 R. Pandharipande, A. Pixton, and D. Zvonkine, Relations on $\overline{\mathcal{M}}_{g, n}$ via 3-spin structures, J. Amer. Math. Soc. 28 (2015), no. 1, 279-309; http://dx.doi.org/10.1090/ S0894-0347-2014-00808-0.

PT14 D. Petersen and O. Tommasi, The Gorenstein conjecture fails for the tautological ring of $\overline{\mathcal{M}}_{2, n}$, Invent. Math. 196 (2014), no. 1, 139-161; http://dx.doi.org/10.1007/s00222-013-0466-z.

Sch63 R. L. E. Schwarzenberger, Jacobians and symmetric products, Illinois J. Math. 7 (1963), 257-268; http://projecteuclid.org/euclid.ijm/1255644637.

Tav11 M. Tavakol, The tautological ring of $M_{1, n}^{c t}$, Ann. Inst. Fourier (Grenoble) 61 (2011), no. 7, 2751-2779; http://dx.doi.org/10.5802/aif.2793.

Tav14 , The tautological ring of the moduli space $M_{2, n}^{r t}$, Int. Math. Res. Not. IMRN 2014 (2014), no. 24, 6661-6683; http://dx.doi.org/10.1093/imrn/rnt178.

Yin12 Q. Yin, On the tautological rings of $\mathcal{M}_{g, 1}$ and its universal Jacobian, arXiv:1206.3783.

Qizheng Yin yin@math.ethz.ch

ETH Zürich, Departement Mathematik, Rämistrasse 101, 8092 Zürich, Switzerland 